\title{
CHARACTERIZATIONS OF HARDY-TYPE, BERGMAN- TYPE AND DIRICHLET-TYPE SPACES ON CERTAIN CLASSES OF COMPLEX-VALUED FUNCTIONS
}

\author{
Shaolin Chen, Antti Rasila and Matti Vuorinen \\ Hengyang Normal University, College of Mathematics and Statistics \\ Hengyang, Hunan 421008, P. R. China; mathechen@126.com \\ Guangdong Technion - Israel Institute of Technology, College of Science \\ 241 Daxue Road, Shantou, Guangdong 515063, P. R. China; antti.rasila@gtiit.edu.cn \\ University of Turku, Department of Mathematics and Statistics \\ Turku 20014, Finland; vuorinen@utu.fi
}

\begin{abstract}
In this paper, we continue our investigation of function spaces on certain classes of complex-valued functions. In particular, we give characterizations on Hardy-type, Bergman-type and Dirichlet-type spaces. Furthermore, we present applications of our results to certain nonlinear PDEs.
\end{abstract}

\section{Introduction and main results}

For a positive integer $n \geq 1$, let $\mathbf{C}^{n}$ denote the complex Euclidean $n$-space. For $z:=\left(z_{1}, \ldots, z_{n}\right)$ and $w=\left(w_{1}, \ldots, w_{n}\right)$ in $\mathbf{C}^{n}$, we let $\bar{z}=\left(\bar{z}_{1}, \ldots, \bar{z}_{n}\right)$, and

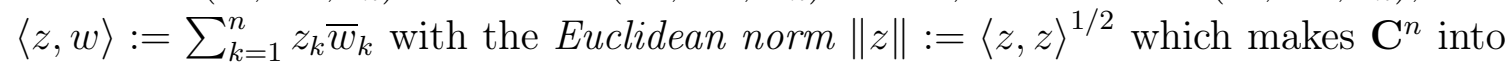
an $n$-dimensional complex Hilbert space. For $a \in \mathbf{C}^{n}$ and $r>0, \mathbf{B}^{n}(a, r)$ denotes the (open) ball of radius $r$ with center $a$. Also, we let $\mathbf{B}^{n}(r):=\mathbf{B}^{n}(0, r)$ and denote the unit ball by $\mathbf{B}^{n}:=\mathbf{B}^{n}(1)$. In particular, let $\mathbf{B}^{1}(r)=\mathbf{D}(r)$ and $\mathbf{D}=\mathbf{B}^{1}$. For a domain $\Omega \subset \mathbf{C}^{n}$ with non-empty boundary, let $d_{\Omega}(z)$ be the Euclidean distance from $z$ to the boundary $\partial \Omega$ of $\Omega$. Moreover, we always use $d(z)$ to denote the Euclidean distance from $z$ to the boundary of $\mathbf{B}^{n}$. We denote by $\mathcal{C}^{m}\left(\mathbf{B}^{n}\right)$ the set of all $m$ time continuously differentiable complex-valued functions $f$ of $\mathbf{B}^{n}$ into $\mathbf{C}$, where $m \in\{0,1, \ldots\}$.

For $k \in\{1, \ldots, n\}, z=\left(z_{1}, \ldots, z_{n}\right) \in \mathbf{C}^{n}$ and $f \in \mathcal{C}^{1}\left(\mathbf{B}^{n}\right)$, we introduce the following notations:

$$
\nabla f=\left(f_{z_{1}}, \ldots, f_{z_{n}}\right), \quad \bar{\nabla} f=\left(f_{\bar{z}_{1}}, \ldots, f_{\bar{z}_{n}}\right) \quad \text { and } \quad D_{f}=(\nabla f, \bar{\nabla} f),
$$

where $f_{z_{k}}=\partial f / \partial z_{k}=1 / 2\left(\partial f / \partial x_{k}-i \partial f / \partial y_{k}\right), f_{\bar{z}_{k}}=\partial f / \partial \bar{z}_{k}=1 / 2\left(\partial f / \partial x_{k}+\right.$ $\left.i \partial f / \partial y_{k}\right)$ and $z_{k}=x_{k}+i y_{k}$, with $x_{k}$ and $y_{k}$ real. Let $\left\|D_{f}\right\|$ be the Hilbert-Schmidt semi-norm given by

$$
\left\|D_{f}\right\|=\left(\|\nabla f\|^{2}+\|\bar{\nabla} f\|^{2}\right)^{1 / 2}
$$

Let $f=u+i v \in \mathcal{C}^{1}\left(\mathbf{B}^{n}\right)$, where $u$ and $v$ are real-valued functions. Then for $z=\left(z_{1}, \ldots, z_{n}\right)=\left(x_{1}+i y_{1}, \ldots, x_{n}+i y_{n}\right) \in \mathbf{B}^{n}$,

$$
\|\nabla f(z)\|+\|\bar{\nabla} f(z)\| \leq\|\nabla u(z)\|+\|\nabla v(z)\|,
$$

where

$$
\nabla u=\left(\frac{\partial u}{\partial x_{1}}, \frac{\partial u}{\partial y_{1}}, \ldots, \frac{\partial u}{\partial x_{n}}, \frac{\partial u}{\partial y_{n}}\right) \quad \text { and } \quad \nabla v=\left(\frac{\partial v}{\partial x_{1}}, \frac{\partial v}{\partial y_{1}}, \ldots, \frac{\partial v}{\partial x_{n}}, \frac{\partial v}{\partial y_{n}}\right)
$$

https://doi.org/10.5186/aasfm.2019.4426

2010 Mathematics Subject Classification: Primary 32A10, 30D55; Secondary 30C65, 58J10.

Key words: Hardy-type space, Bergman-type space, Dirichlet-type space. 
Note that the converse of (1.1) is not always true (see [5]).

Generalized Hardy spaces. For $p \in(0, \infty]$, the generalized Hardy space $\mathcal{H}_{g}^{p}\left(\mathbf{B}^{n}\right)$ consists of measurable functions $f: \mathbf{B}^{n} \rightarrow \mathbf{C}$ such that $M_{p}(r, f)$ exists for all $r \in(0,1)$ and $\|f\|_{p}<\infty$, where

$$
\|f\|_{p}=\left\{\begin{array}{ll}
\sup _{0<r<1} M_{p}(r, f), & \text { if } p \in(0, \infty), \\
\sup _{z \in \mathbf{B}^{n}}|f(z)|, & \text { if } p=\infty,
\end{array} \quad M_{p}(r, f)=\left(\int_{\partial \mathbf{B}^{n}}|f(r \zeta)|^{p} d \sigma(\zeta)\right)^{1 / p}\right.
$$

and $d \sigma$ denotes the normalized Lebesgue surface measure in $\partial \mathbf{B}^{n}$.

There are numerous characterizations of the classical analytic Hardy spaces in the literature, see for example [12, 17, 18, 21, 22, 27]. But, to our knowledge, there are few analogous results for general complex-valued functions. In this paper, we give the following characterization of a class of complex-valued functions $f$ in Hardy-type spaces.

Theorem 1. For $p \geq 2$, let $f \in \mathcal{C}^{2}\left(\mathbf{B}^{n}\right)$ with $\operatorname{Re}(f \overline{\Delta f}) \geq 0$. Then,

$$
\int_{\mathbf{B}^{n}} d(z) \Delta\left(|f(z)|^{p}\right) d V_{N}(z)<\infty
$$

if and only if $f \in \mathcal{H}_{g}^{p}\left(\mathbf{B}^{n}\right)$, where $d V_{N}$ denotes the normalized Lebesgue volume measure in $\mathbf{B}^{n}$ and $\Delta$ is the usual complex Laplacian operator

$$
\Delta:=4 \sum_{k=1}^{n} \frac{\partial^{2}}{\partial z_{k} \partial \bar{z}_{k}}=\sum_{k=1}^{n}\left(\frac{\partial^{2}}{\partial x_{k}^{2}}+\frac{\partial^{2}}{\partial y_{k}^{2}}\right)
$$

for $z=\left(z_{1}, \ldots, z_{n}\right)=\left(x_{1}+i y_{1}, \ldots, x_{n}+i y_{n}\right) \in \mathbf{B}^{n}$.

Yukawa PDE. Let $\tau, \eta: \mathbf{B}^{n} \rightarrow[0, \infty)$ be continuous and $f=u+i v \in \mathcal{C}^{2}\left(\mathbf{B}^{n}\right)$, where $u$ and $v$ are real-valued functions in $\mathbf{B}^{n}$. The nonlinear elliptic partial differential equation (PDE) of the form

$$
\Delta f(z)=\tau(z) f(z)+\eta(z) \operatorname{Re}(f(z))
$$

is called the non-homogeneous Yukawa PDE, where $z \in \mathbf{B}^{n}$. If $\tau$ in (1.2) is a positive constant function and $\eta \equiv 0$, then we have the usual Yukawa PDE. This equation arose from the work of the Japanese Nobel physicist Hideki Yukawa, who used it to describe the nuclear potential of a point charge as $e^{-\sqrt{\tau} r} / r$ (cf. $[1,3,7,9,10,11,16$, 30, 34]).

As an application of Theorem 1, we obtain the following result.

Corollary 1.1. For $p \geq 2$, let $f \in \mathcal{C}^{2}\left(\mathbf{B}^{n}\right)$ satisfying (1.2). Then,

$$
\int_{\mathbf{B}^{n}} d(z) \Delta\left(|f(z)|^{p}\right) d V_{N}(z)<\infty
$$

if and only if $f \in \mathcal{H}_{g}^{p}\left(\mathbf{B}^{n}\right)$.

A continuous increasing function $\omega:[0, \infty) \rightarrow[0, \infty)$ with $\omega(0)=0$ is called a majorant if $\omega(t) / t$ is non-increasing for $t>0$ (cf. [14, 15, 25, 26]). Given a subset $\Omega$ of $\mathbf{C}^{n}$, a function $f: \Omega \rightarrow \mathbf{C}$ is said to belong to the Lipschitz space $L_{\omega}(\Omega)$ if there is a positive constant $C$ such that

$$
|f(z)-f(w)| \leq C \omega(|z-w|) \quad \text { for all } z, w \in \Omega .
$$


A classical result of Hardy and Littlewood asserts that if $p \in(0, \infty], \alpha \in(1, \infty)$ and $f$ is an analytic function in $\mathbf{D}$, then (cf. [12, 21, 22])

$$
M_{p}\left(r, f^{\prime}\right)=O\left(\left(\frac{1}{1-r}\right)^{\alpha}\right) \quad \text { as } r \rightarrow 1
$$

if and only if

$$
M_{p}(r, f)=O\left(\left(\log \frac{1}{1-r}\right)^{\alpha-1}\right) \quad \text { as } r \rightarrow 1 .
$$

In [17], via the closed graph theorem, Girela, Pavlović and Peláez refined the above result for the case $\alpha=1$ as follows.

Theorem A. [17, Theorem 1.1] Let $p \in(2, \infty)$. For $r \in(0,1)$, if $f$ is analytic in D such that

$$
M_{p}\left(r, f^{\prime}\right)=O\left(\frac{1}{1-r}\right) \quad \text { as } r \rightarrow 1
$$

then

$$
M_{p}(r, f)=O\left(\left(\log \frac{1}{1-r}\right)^{\frac{1}{2}}\right) \quad \text { as } r \rightarrow 1
$$

and the exponent $1 / 2$ is sharp.

Theorem A gives an affirmative answer to the open problem in [18, p. 464, Equation (26)]. For related investigations on this topic, we refer to [3, 4, 7, 32].

Next we study the relationship between the integral means of solutions to the equation (1.2) and those of their second order partial derivative. Our result is given as follows.

Theorem 2. Let $\omega$ be a majorant and $f \in \mathcal{C}^{2}\left(\mathbf{B}^{n}\right)$ satisfying (1.2) with $\eta+\tau<$ $4 n / p$, where $\tau$ and $\eta$ are nonnegative constant functions. For $p \geq 2$ and $r \in(0,1)$, if

$$
M_{p}\left(r, D_{f}^{*}\right) \leq M^{*} \omega\left(\frac{1}{1-r}\right)
$$

then

$$
M_{p}\left(r, D_{f}\right) \leq \sqrt{M_{2}^{*}}\left[\left\|D_{f}(0)\right\|^{2}+M_{1}^{*} \int_{0}^{1} \omega\left(\frac{1}{1-r t}\right) d t\right]^{\frac{1}{2}}
$$

and $f \in \mathcal{H}_{g}^{p}\left(\mathbf{B}^{n}\right)$, where $M^{*}$ is a positive constant,

$$
\begin{gathered}
D_{f}^{*}=\left[\sum_{j=1}^{n} \sum_{k=1}^{n}\left(\left|f_{z_{k} z_{j}}\right|^{2}+\left|f_{z_{k} \bar{z}_{j}}\right|^{2}+\left|f_{\bar{z}_{k} z_{j}}\right|^{2}+\left|f_{\bar{z}_{k} \bar{z}_{j}}\right|^{2}\right)\right]^{\frac{1}{2}}, \\
M_{1}^{*}=2 p(2 p-3)\left(M^{*}\right)^{2} \omega(1) \text { and } M_{2}^{*}=1 /[1-p(\eta+\tau) /(4 n)] .
\end{gathered}
$$

In particular, by taking $\omega(t)=t$ in Theorem 2, we obtain the following result.

Corollary 1.2. Let $p \geq 2$ and $f \in \mathcal{C}^{2}\left(\mathbf{B}^{n}\right)$ satisfying (1.2) with $\eta+\tau<4 n / p$, where $\tau$ and $\eta$ are nonnegative constant functions. For $r \in(0,1)$, if

$$
M_{p}\left(r, D_{f}^{*}\right)=O\left(\frac{1}{1-r}\right) \quad \text { as } r \rightarrow 1,
$$

then

$$
M_{p}\left(r, D_{f}\right)=O\left(\left(\log \frac{1}{1-r}\right)^{\frac{1}{2}}\right) \quad \text { as } r \rightarrow 1
$$

and $f \in \mathcal{H}_{g}^{p}\left(\mathbf{B}^{n}\right)$. 
Dirichlet-type spaces and Bergman-type spaces. For $\nu, \mu, t \in \mathbf{R}$,

$$
\mathcal{D}_{f}(\nu, \mu, t)=\int_{\mathbf{B}^{n}} d^{\nu}(z)|f(z)|^{\mu}\left\|D_{f}(z)\right\|^{t} d V_{N}(z)<\infty
$$

is called Dirichlet-type energy integral of the complex-valued function $f$ (cf. $[1,2,7$, $16,17,19,31,32,33,34])$. In particular, for $\nu \geq 0, \mu=0$ and $0<t<\infty$, we use $\mathcal{D}_{\nu, t}\left(\mathbf{B}^{n}\right)$ to denote the Dirichlet-type space consisting of all $f \in \mathcal{C}^{1}\left(\mathbf{B}^{n}\right)$ with the norm

$$
\|f\|_{\mathcal{D}_{\nu, t}}=|f(0)|+\left(\mathcal{D}_{f}(\nu, 0, t)\right)^{1 / t}<\infty .
$$

Moreover, for $\nu>-1,0<\mu<\infty$ and $t=0$, we denote by $b_{\nu, \mu}\left(\mathbf{B}^{n}\right)$ the Bergman-type space consisting of all $f \in \mathcal{C}^{0}\left(\mathbf{B}^{n}\right)$ with the norm

$$
\|f\|_{b_{\nu, \mu}}=|f(0)|+\left(\mathcal{D}_{f}(\nu, \mu, 0)\right)^{1 / \mu}<\infty .
$$

We refer to $[13,17,19,20,26,28,35]$ for basic characterizations of analytic (or harmonic) Bergman-type spaces and Dirichlet-type spaces. Again, for general complex-valued functions, very little related research can be found from the literature. The following is a characterization of a class of complex-valued functions $f$ in Bergman-type spaces.

Theorem 3. Let $f \in \mathcal{C}^{2}\left(\mathbf{B}^{n}\right)$ with $\operatorname{Re}(f \overline{\Delta f}) \geq 0$. Then, for $p \geq 2$ and $\alpha \geq 2$,

$$
\int_{\mathbf{B}^{n}}\left(1-|z|^{2}\right)^{\alpha} \Delta\left(|f(z)|^{p}\right) d V_{N}(z)<\infty
$$

if and only if $f \in b_{\alpha-2, p}\left(\mathbf{B}^{n}\right)$.

The following result easily follows from Theorem 3 .

Corollary 1.3. Let $f \in \mathcal{C}^{2}\left(\mathbf{B}^{n}\right)$ satisfy (1.2). Then, for $p \geq 2$ and $\alpha \geq 2$,

$$
\int_{\mathbf{B}^{n}}\left(1-|z|^{2}\right)^{\alpha} \Delta\left(|f(z)|^{p}\right) d V_{N}(z)<\infty
$$

if and only if $f \in b_{\alpha-2, p}\left(\mathbf{B}^{n}\right)$.

Definition 1. For $m \in\{2,3, \ldots\}$, we denote by $\mathcal{H Z}_{m}\left(\mathbf{B}^{n}\right)$ the class of all functions $f \in \mathcal{C}^{m}\left(\mathbf{B}^{n}\right)$ satisfying Heinz's nonlinear differential inequality (cf. [23])

$$
|\Delta f(z)| \leq a(z)\left\|D_{f}(z)\right\|+b(z)|f(z)|+c(z),
$$

where $a(z), b(z)$ and $c(z)$ are real-valued nonnegative continuous functions in $\mathbf{B}^{n}$.

Theorem 4. Let $M$ be a nonnegative constant and $f \in \mathcal{H Z}_{3}\left(\mathbf{B}^{n}\right) \cap \mathcal{D}_{\gamma, \alpha}\left(\mathbf{B}^{n}\right)$ with $\operatorname{Re}(f \overline{\Delta f}) \geq 0$ and $\operatorname{Re}\left\{\sum_{k=1}^{n}\left[\overline{f_{z_{k}}}(\Delta f)_{z_{k}}+\overline{f_{\bar{z}_{k}}}(\Delta f)_{\bar{z}_{k}}\right]\right\} \geq 0$, where $2 \leq \alpha \leq 2 n$, $\gamma>0, \sup _{z \in \mathbf{B}^{n}} a(z)<\infty, \sup _{z \in \mathbf{B}^{n}} b(z)<\infty$ and $c(z) \leq M(d(z))^{-q}$. Then for $p \geq 2$,

$$
\int_{\mathbf{B}^{n}}(d(z))^{p q} \Delta\left(|f(z)|^{p}\right) d V_{N}(z)<\infty
$$

where $q=(2 n+\gamma) / \alpha-1$.

The result given below is a consequence of Theorem 4 .

Corollary 1.4. For $2 \leq \alpha \leq 2 n$ and $\gamma>0$, let $f \in \mathcal{H Z}_{3}\left(\mathbf{B}^{n}\right) \cap \mathcal{D}_{\gamma, \alpha}\left(\mathbf{B}^{n}\right)$ satisfying (1.2), where $\tau$ and $\eta$ are nonnegative constant functions. Then for $p \geq 2$,

$$
\int_{\mathbf{B}^{n}}(d(z))^{p q} \Delta\left(|f(z)|^{p}\right) d V_{N}(z)<\infty
$$

where $q=(2 n+\gamma) / \alpha-1$. 
Proof. By elementary calculations, we see that if $f$ is a solution to (1.2), then $f$ satisfies Heinz's nonlinear differential inequality. Hence Corollary 1.4 follows from (2.8), (2.9) and Theorem 4.

By Corollaries 1.1, 1.3 and 1.4, we get

Corollary 1.5. For $2 \leq \alpha \leq 2 n$ and $\gamma>0$, let $q=(2 n+\gamma) / \alpha-1$ and let $f \in \mathcal{H Z}_{3}\left(\mathbf{B}^{n}\right) \cap \mathcal{D}_{\gamma, \alpha}\left(\mathbf{B}^{n}\right)$ satisfy (1.2), where $\tau$ and $\eta$ are nonnegative constant functions.

(1) If $p=\frac{1}{q} \geq 2$, then $f \in \mathcal{H}_{g}^{p}\left(\mathbf{B}^{n}\right)$;

(2) If $p \geq 2$ and $p q \geq 2$, then $f \in b_{p q-2, p}\left(\mathbf{B}^{n}\right)$.

Definition 2. For $p \geq 2, t_{1}>0, t_{2}>0$ and $m \in\{2,3, \ldots\}$, we denote by $\mathcal{I} \mathcal{H} \mathcal{Z}_{m}^{t_{1}, t_{2}}\left(\mathbf{B}^{n}\right)$ the class of all functions $f \in \mathcal{C}^{m}\left(\mathbf{B}^{n}\right)$ satisfying the inverse Heinz's nonlinear differential inequality

$$
\Delta\left(|f(z)|^{p}\right) \geq a_{1}(z)\left\|D_{f}(z)\right\|^{t_{1}}+b_{1}(z)|f(z)|^{t_{2}}+c_{1}(z),
$$

where $a_{1}(z), b_{1}(z)$ and $c_{1}(z)$ are real-valued nonnegative continuous functions in $\mathbf{B}^{n}$.

Theorem 5. Let $f \in \mathcal{I} \mathcal{H} \mathcal{Z}_{2}^{t_{1}, t_{2}}\left(\mathbf{B}^{n}\right) \cap \mathcal{H}_{g}^{p}\left(\mathbf{B}^{n}\right)$, where $\inf _{z \in \mathbf{B}^{n}} a_{1}(z)+\inf _{z \in \mathbf{B}^{n}} b_{1}(z)$ $>0$ and $\inf _{z \in \mathbf{B}^{n}} c_{1}(z) \geq 0$.

(1) If $\inf _{z \in \mathbf{B}^{n}} a_{1}(z)>0$, then $f \in \mathcal{D}_{1, t_{1}}\left(\mathbf{B}^{n}\right)$;

(2) If $\inf _{z \in \mathbf{B}^{n}} b_{1}(z)>0$, then $f \in b_{1, t_{2}}\left(\mathbf{B}^{n}\right)$.

For $k \in\{1, \ldots, n\}$, let $\lambda_{k} \in \mathbf{R}$ be a constant and let $f \in \mathcal{C}^{1}\left(\mathbf{B}^{n}\right)$ satisfy the following nonlinear PDE,

$$
\frac{\partial f}{\partial \bar{z}_{k}}=\lambda_{k}|f|^{\alpha}
$$

where $\alpha \geq 0$. If, for each $k \in\{1, \ldots, n\}, \lambda_{k}=0$, then $f$ is holomorphic. Moreover, if $\alpha=0$, then $f$ is pluriharmonic (cf. $[6,29]$ ). It has attracted the attention of many authors when $n=\lambda_{1}=1$ and $\alpha \in(0,1)$ (cf. [2, 8, 24]).

Corollary 1.6. For $\sum_{k=1}^{n} \lambda_{k}^{2} \neq 0, \alpha \geq 0$ and $p>\max \left\{2,(\alpha-2)^{2} / 4\right\}$, if $f \in$ $\mathcal{H}_{g}^{p}\left(\mathbf{B}^{n}\right) \cap \mathcal{C}^{2}\left(\mathbf{B}^{n}\right)$ satisfies (1.3), then $f \in b_{1, \vartheta}\left(\mathbf{B}^{n}\right)$, where $\vartheta=p+2 \alpha-2$.

The proofs of Theorems 1 and 2 will be presented in Section 2, and the proofs of Theorems 3, 4, 5 and Corollary 1.6 will be given in Section 3.

\section{Hardy-type spaces and applications to pdes}

We start this section by recalling the following result.

Theorem B. [27] Let $g$ be a function of class $\mathcal{C}^{2}\left(\mathbf{B}^{n}\right)$. Then, for $r \in(0,1)$,

$$
\int_{\partial \mathbf{B}^{n}} g(r \zeta) d \sigma(\zeta)=g(0)+\int_{\mathbf{B}^{n}(r)} \Delta g(z) G_{2 n}(z, r) d V_{N}(z)
$$

where

$$
G_{2 n}(z, r)= \begin{cases}\frac{|z|^{2(1-n)}-r^{2(1-n)}}{4 n(n-1)}, & \text { if } n \geq 2, \\ \frac{1}{2} \log \frac{r}{|z|}, & \text { if } n=1 .\end{cases}
$$

Lemma 1. Let $p \geq 2$ and $f \in \mathcal{C}^{2}\left(\mathbf{B}^{n}\right)$ with $\operatorname{Re}(f \overline{\Delta f}) \geq 0$. Then $M_{p}^{p}(r, f)$ is increasing with respect to $r \in(0,1)$. 
Proof. Case 1. Let $p \in[4, \infty)$. By elementary calculations, we get

$$
\Delta\left(|f|^{p}\right)=p(p-2)|f|^{p-4} \sum_{k=1}^{n}\left|f_{z_{k}} \bar{f}+\overline{f_{\bar{z}_{k}}} f\right|^{2}+2 p|f|^{p-2}\left\|D_{f}\right\|^{2}+p|f|^{p-2} \operatorname{Re}(f \overline{\Delta f}) \geq 0
$$

which implies that, for $p \in[4, \infty), M_{p}^{p}(r, f)$ is increasing in $(0,1)$.

Case 2. Let $p \in[2,4)$. For $m \in\{1,2, \ldots\}$, let $T_{m}^{p}=\left(|f|^{2}+\frac{1}{m}\right)^{\frac{p}{2}}$. By computations, we have

$$
\begin{aligned}
\Delta\left(T_{m}^{p}\right)= & 4 \sum_{k=1}^{n} \frac{\partial^{2}}{\partial z_{k} \partial \bar{z}_{k}}\left(T_{m}^{p}\right)=4 \sum_{k=1}^{n}\left(T_{m}^{p}\right)_{z_{k} \bar{z}_{k}} \\
= & p(p-2)\left(|f|^{2}+\frac{1}{m}\right)^{\frac{p}{2}-2} \sum_{k=1}^{n}\left|f_{z_{k}} \bar{f}+\overline{f_{\bar{z}_{k}}} f\right|^{2} \\
& +2 p\left(|f|^{2}+\frac{1}{m}\right)^{\frac{p}{2}-1}\left\|D_{f}\right\|^{2}+p\left(|f|^{2}+\frac{1}{m}\right)^{\frac{p}{2}-1} \operatorname{Re}(f \overline{\Delta f}) .
\end{aligned}
$$

Let $Q_{m}=\Delta\left(T_{m}^{p}\right)$. It is not difficult to show that, for $r \in(0,1), Q_{m}$ is integrable in $\mathbf{B}^{n}(r)$ and $0<Q_{m} \leq \Lambda_{f}$, where

$$
\begin{aligned}
\Lambda_{f}= & p(p-2)|f|^{p-2} \sum_{k=1}^{n}\left(\left|f_{z_{k}}\right|+\left|f_{\bar{z}_{k}}\right|\right)^{2}+2 p\left(|f|^{2}+1\right)^{\frac{p}{2}-1}\left\|D_{f}\right\|^{2} \\
& +p\left(|f|^{2}+1\right)^{\frac{p}{2}-1} \operatorname{Re}(f \overline{\Delta f})
\end{aligned}
$$

and $\Lambda_{f}$ is integrable in $\mathbf{B}^{n}(r)$.

By using Theorem B and Lebesgue's dominated convergence theorem, we get

$$
\begin{aligned}
\lim _{m \rightarrow \infty} r^{2 n-1} \frac{d}{d r} M_{p}^{p}\left(r, T_{m}\right)= & \frac{1}{2 n} \lim _{m \rightarrow \infty} \int_{\mathbf{B}^{n}(r)} Q_{m} d V_{N}=\frac{1}{2 n} \int_{\mathbf{B}^{n}(r)} \lim _{m \rightarrow \infty} Q_{m} d V_{N} \\
= & \frac{1}{2 n} \int_{\mathbf{B}^{n}(r)}\left[p(p-2)|f|^{p-4} \sum_{k=1}^{n}\left|f \overline{f_{z_{k}}}+\bar{f} f_{\bar{z}_{k}}\right|^{2}\right. \\
& \left.+2 p|f|^{p-2}\left\|D_{f}\right\|^{2}+p|f|^{p-2} \operatorname{Re}(f \overline{\Delta f})\right] d V_{N} \\
= & r^{2 n-1} \frac{d}{d r} M_{p}^{p}(r, f) \geq 0,
\end{aligned}
$$

which implies that $M_{p}^{p}(r, f)$ is increasing in $r$ on $(0,1)$ for $p \in[2,4)$.

By using Theorem B and a similar argument as in the proof of Lemma 1, we obtain the following result.

Lemma 2. Let $p \geq 2$ and $f \in \mathcal{C}^{2}\left(\mathbf{B}^{n}\right)$ with $\operatorname{Re}(f \overline{\Delta f}) \geq 0$. Then, for $r \in(0,1)$,

$$
M_{p}^{p}(r, f)=|f(0)|^{p}+\int_{\mathbf{B}^{n}(r)} \Delta\left(|f(z)|^{p}\right) G_{2 n}(z, r) d V_{N}(z)
$$

where $G_{2 n}$ is the function defined in Theorem B. 
Proof of Theorem 1. Case 1. Let $n \geq 2$. We first prove the necessity. For a fixed positive constant $r_{0} \in(0,1)$, let $r \in\left(r_{0}, 1\right)$. Then, by Lemma 2 , we have

$$
\begin{aligned}
M_{p}^{p}(r, f)= & |f(0)|^{p}+\int_{\mathbf{B}^{n}(r)} \Delta\left(|f(z)|^{p}\right) G_{2 n}(z, r) d V_{N}(z) \\
= & |f(0)|^{p}+\frac{1}{4 n(n-1)} \int_{\mathbf{B}^{n}(r) \backslash \mathbf{B}^{n}\left(r_{0}\right)}\left(|z|^{2(1-n)}-r^{2(1-n)}\right) \Delta\left(|f(z)|^{p}\right) d V_{N}(z) \\
& +\frac{1}{4 n(n-1)} \int_{\mathbf{B}^{n}\left(r_{0}\right)}\left(|z|^{2(1-n)}-r^{2(1-n)}\right) \Delta\left(|f(z)|^{p}\right) d V_{N}(z) .
\end{aligned}
$$

Since $\Delta\left(|f|^{p}\right) \geq 0$,

$$
\begin{aligned}
\infty & >2 n \int_{\partial \mathbf{B}^{n}} \int_{0}^{r_{0}}\left(\rho-\rho^{2 n-1}\right) \Delta\left(|f(\rho \zeta)|^{p}\right) d \rho d \sigma(\zeta) \\
& \geq 2 n \int_{\partial \mathbf{B}^{n}} \int_{0}^{r_{0}}\left(\rho-r^{2(1-n)} \rho^{2 n-1}\right) \Delta\left(|f(\rho \zeta)|^{p}\right) d \rho d \sigma(\zeta) \\
& =\int_{\mathbf{B}^{n}\left(r_{0}\right)}\left(|z|^{2(1-n)}-r^{2(1-n)}\right) \Delta\left(|f(z)|^{p}\right) d V_{N}(z)
\end{aligned}
$$

and

$$
\begin{aligned}
& \int_{\mathbf{B}^{n}(r) \backslash \mathbf{B}^{n}\left(r_{0}\right)}\left(|z|^{2(1-n)}-r^{2(1-n)}\right) \Delta\left(|f(z)|^{p}\right) d V_{N}(z) \\
& =\int_{\mathbf{B}^{n}(r) \backslash \mathbf{B}^{n}\left(r_{0}\right)} \frac{(r-|z|)\left(\sum_{k=0}^{2 n-3} r^{2 n-3-k}|z|^{k}\right)}{|z|^{2 n-2} r^{2 n-2}} \Delta\left(|f(z)|^{p}\right) d V_{N}(z) \\
& \leq \frac{(2 n-2)}{r_{0}^{4 n-4}} \int_{\mathbf{B}^{n}(r) \backslash \mathbf{B}^{n}\left(r_{0}\right)}(r-|z|) \Delta\left(|f(z)|^{p}\right) d V_{N}(z) \\
& \leq \frac{(2 n-2)}{r_{0}^{4 n-4}} \int_{\mathbf{B}^{n}} d(z) \Delta\left(|f(z)|^{p}\right) d V_{N}(z)<\infty .
\end{aligned}
$$

By (2.1) and Lemma 1, we see that the limit

$$
\lim _{r \rightarrow 1-} M_{p}(r, f)
$$

exists. Hence $f \in \mathcal{H}_{g}^{p}\left(\mathbf{B}^{n}\right)$.

Next we prove the sufficiency. Applying (2.1), (2.2) and $f \in \mathcal{H}_{g}^{p}\left(\mathbf{B}^{n}\right)$, we observe that

$$
\begin{aligned}
\infty & >\int_{\mathbf{B}^{n}(r) \backslash \mathbf{B}^{n}\left(r_{0}\right)}\left(|z|^{2(1-n)}-r^{2(1-n)}\right) \Delta\left(|f(z)|^{p}\right) d V_{N}(z) \\
& =\int_{\mathbf{B}^{n}(r) \backslash \mathbf{B}^{n}\left(r_{0}\right)} \frac{(r-|z|)\left(\sum_{k=0}^{2 n-3} r^{2 n-3-k}|z|^{k}\right)}{|z|^{2 n-2} r^{2 n-2}} \Delta\left(|f(z)|^{p}\right) d V_{N}(z) \geq I(r),
\end{aligned}
$$

which, together with the monotonicity of $I(r)$ on $r \in\left[r_{0}, 1\right)$, yields that

$$
\lim _{r \rightarrow 1-} \int_{\mathbf{B}^{n}(r) \backslash \mathbf{B}^{n}\left(r_{0}\right)}(r-|z|) \Delta\left(|f(z)|^{p}\right) d V_{N}(z)
$$

exists, where

$$
I(r)=(2 n-2) r_{0}^{2 n-3} \int_{\mathbf{B}^{n}(r) \backslash \mathbf{B}^{n}\left(r_{0}\right)}(r-|z|) \Delta\left(|f(z)|^{p}\right) d V_{N}(z) .
$$


Therefore,

$$
\int_{\mathbf{B}^{n}} d(z) \Delta\left(|f(z)|^{p}\right) d V_{N}(z)<\infty
$$
Since

Case 2. Let $n=1$. In this case, we also first prove the necessity. Fix $r \in(0,1)$.

$$
\lim _{|z| \rightarrow r} \frac{\log r-\log |z|}{r-|z|}=\frac{1}{r}
$$

we see that there exists $r_{0} \in(0, r)$ such that

$$
\frac{1}{2 r} \leq \frac{\log r-\log |z|}{r-|z|} \leq \frac{3}{2 r}
$$

for $r_{0} \leq|z|<r$. It is not difficult to see that, for $|z| \leq r<1$,

$$
\frac{r-|z|}{r} \leq 1-|z|
$$

Because

$$
\lim _{\rho \rightarrow 0+} \rho \log \frac{1}{\rho}=0
$$

it follows that

$$
\begin{aligned}
\int_{\mathbf{D}\left(r_{0}\right)} \Delta\left(|f(z)|^{p}\right) \log \frac{r}{|z|} d A(z) & =\int_{\mathbf{D}\left(r_{0}\right)} \Delta\left(|f(z)|^{p}\right) \log \frac{1}{|z|} d A(z) \\
& =\frac{1}{\pi} \int_{0}^{2 \pi} \int_{0}^{r_{0}} \Delta\left(\left|f\left(\rho e^{i \theta}\right)\right|^{p}\right) \rho \log \frac{1}{\rho} d \theta<\infty
\end{aligned}
$$

where $d A$ denotes the normalized area measure in $\mathbf{D}$.

By (2.3), (2.4), (2.5), Lemmas 1 and 2, we see that

$$
\begin{aligned}
& M_{p}^{p}(r, f)=|f(0)|^{p}+\frac{1}{2} \int_{\mathbf{D}(r)} \Delta\left(|f(z)|^{p}\right) \log \frac{r}{|z|} d A(z) \\
& =|f(0)|^{p}+\frac{1}{2} \int_{\mathbf{D}\left(r_{0}\right)} \Delta\left(|f(z)|^{p}\right) \log \frac{r}{|z|} d A(z)+\frac{1}{2} \int_{\mathbf{D}(r) \backslash \mathbf{D}\left(r_{0}\right)} \Delta\left(|f(z)|^{p}\right) \log \frac{r}{|z|} d A(z) \\
& \leq|f(0)|^{p}+\frac{1}{2} \int_{\mathbf{D}\left(r_{0}\right)} \Delta\left(|f(z)|^{p}\right) \log \frac{r}{|z|} d A(z)+\frac{3}{4} \int_{\mathbf{D}(r) \backslash \mathbf{D}\left(r_{0}\right)} \Delta\left(|f(z)|^{p}\right) \frac{(r-|z|)}{r} d A(z) \\
& \leq|f(0)|^{p}+\frac{1}{2} \int_{\mathbf{D}\left(r_{0}\right)} \Delta\left(|f(z)|^{p}\right) \log \frac{r}{|z|} d A(z)+\frac{3}{4} \int_{\mathbf{D} \backslash \mathbf{D}\left(r_{0}\right)} \Delta\left(|f(z)|^{p}\right) d(z) d A(z)<\infty,
\end{aligned}
$$

which implies that the limit

$$
\lim _{r \rightarrow 1-} M_{p}(r, f)
$$

exists. Hence $f \in \mathcal{H}_{g}^{p}\left(\mathbf{B}^{n}\right)$.

Now we prove the sufficiency. By (2.3), we have

$$
\begin{aligned}
\int_{\mathbf{D}(r) \backslash \mathbf{D}\left(r_{0}\right)} \Delta\left(|f(z)|^{p}\right) \log \frac{r}{|z|} d A(z) & \geq \frac{1}{2 r} \int_{\mathbf{D}(r) \backslash \mathbf{D}\left(r_{0}\right)} \Delta\left(|f(z)|^{p}\right)(r-|z|) d A(z) \\
& \geq \frac{I^{*}(r)}{2},
\end{aligned}
$$

where

$$
I^{*}(r)=\int_{\mathbf{D}(r) \backslash \mathbf{D}\left(r_{0}\right)} \Delta\left(|f(z)|^{p}\right)(r-|z|) d A(z) .
$$


By (2.6), Lemmas 1 and 2, we have

$$
\begin{aligned}
& M_{p}^{p}(r, f)=|f(0)|^{p}+\frac{1}{2} \int_{\mathbf{D}(r)} \Delta\left(|f(z)|^{p}\right) \log \frac{r}{|z|} d A(z) \\
& =|f(0)|^{p}+\frac{1}{2} \int_{\mathbf{D}\left(r_{0}\right)} \Delta\left(|f(z)|^{p}\right) \log \frac{r}{|z|} d A(z)+\frac{1}{2} \int_{\mathbf{D}(r) \backslash \mathbf{D}\left(r_{0}\right)} \Delta\left(|f(z)|^{p}\right) \log \frac{r}{|z|} d A(z) \\
& \geq|f(0)|^{p}+\frac{1}{2} \int_{\mathbf{D}\left(r_{0}\right)} \Delta\left(|f(z)|^{p}\right) \log \frac{r}{|z|} d A(z)+\frac{1}{4} I^{*}(r),
\end{aligned}
$$

which yields that $I^{*}(r)<\infty$. Since $I^{*}(r)$ is increasing on $r$, we see that

$$
\lim _{r \rightarrow 1-} I^{*}(r)
$$

exists. Then

$$
\int_{\mathbf{D}} d(z) \Delta\left(|f(z)|^{p}\right) d A(z)<\infty
$$

concluding the proof of the theorem.

Lemma 3. Let $f \in \mathcal{C}^{3}\left(\mathbf{B}^{n}\right)$ and $\operatorname{Re}\left\{\sum_{k=1}^{n}\left[\overline{f_{z_{k}}}(\Delta f)_{z_{k}}+\overline{f_{\bar{z}_{k}}}(\Delta f)_{\bar{z}_{k}}\right]\right\} \geq 0$. Then, for $\alpha \geq 2,\left\|D_{f}\right\|^{\alpha}$ is subharmonic in $\mathbf{B}^{n}$.

Proof. First we consider the case $\alpha \in[4, \infty)$. Since

$$
\begin{aligned}
\Delta\left(\left\|D_{f}\right\|^{\alpha}\right)= & \alpha(\alpha-2)\left\|D_{f}\right\|^{\alpha-4}\left|\sum_{j=1}^{n} \sum_{k=1}^{n}\left(f_{z_{k} z_{j}} \overline{f_{z_{k}}}+\overline{f_{z_{k} \bar{z}_{j}}} f_{z_{k}}+f_{\bar{z}_{k} z_{j}} \overline{f_{\bar{z}_{k}}}+\overline{f_{\bar{z}_{k} \bar{z}_{j}}} f_{\bar{z}_{k}}\right)\right|^{2} \\
& +2 \alpha\left\|D_{f}\right\|^{\alpha-2} \sum_{j=1}^{n} \sum_{k=1}^{n}\left(\left|f_{z_{j} z_{k}}\right|^{2}+\left|f_{z_{j} \bar{z}_{k}}\right|^{2}+\left|f_{\bar{z}_{j} z_{k}}\right|^{2}+\left|f_{\bar{z}_{j} \bar{z}_{k}}\right|^{2}\right) \\
& +\alpha\left\|D_{f}\right\|^{\alpha-2} \operatorname{Re}\left\{\sum_{k=1}^{n}\left[\overline{f_{z_{k}}}(\Delta f)_{z_{k}}+\overline{f_{\bar{z}_{k}}}(\Delta f)_{\bar{z}_{k}}\right]\right\} \geq 0,
\end{aligned}
$$

we see that, for $\alpha \in[4, \infty),\left\|D_{f}\right\|^{\alpha}$ is subharmonic in $\mathbf{B}^{n}$.

Next we deal with the case $\alpha \in[2,4)$. In this case, for $m \in\{1,2, \ldots\}$, we let $F_{m}^{\alpha}=\left(\left\|D_{f}\right\|^{2}+\frac{1}{m}\right)^{\frac{\alpha}{2}}$. Then, by elementary computations, we have

$$
\begin{aligned}
\Delta\left(F_{m}^{\alpha}\right)= & 4 \sum_{j=1}^{n}\left(F_{m}^{\alpha}\right)_{z_{k} \bar{z}_{k}}=4 \sum_{j=1}^{n} \frac{\partial^{2}}{\partial z_{j} \partial \bar{z}_{j}}\left\{\left[\frac{1}{m}+\sum_{k=1}^{n}\left(f_{z_{k}} \overline{f_{z_{k}}}+f_{\bar{z}_{k}} \overline{f_{\bar{z}_{k}}}\right)\right]^{\frac{\alpha}{2}}\right\} \\
= & \alpha(\alpha-2)\left(\left\|D_{f}\right\|^{2}+\frac{1}{m}\right)^{\frac{\alpha}{2}-2}\left[\sum_{j=1}^{n} \frac{\partial}{\partial z_{j}}\left(\left\|D_{f}\right\|^{2}\right)\right]\left[\sum_{j=1}^{n} \frac{\partial}{\partial \bar{z}_{j}}\left(\left\|D_{f}\right\|^{2}\right)\right] \\
& +2 \alpha\left(\left\|D_{f}\right\|^{2}+\frac{1}{m}\right)^{\frac{\alpha}{2}-1} \sum_{j=1}^{n} \sum_{k=1}^{n}\left(\left|f_{z_{j} z_{k}}\right|^{2}+\left|f_{z_{j} \bar{z}_{k}}\right|^{2}+\left|f_{\bar{z}_{j} z_{k}}\right|^{2}+\left|f_{\bar{z}_{j} \bar{z}_{k}}\right|^{2}\right) \\
& +\alpha\left(\left\|D_{f}\right\|^{2}+\frac{1}{m}\right)^{\frac{\alpha}{2}-1} \operatorname{Re}\left\{\sum_{k=1}^{n}\left[\overline{f_{z_{k}}}(\Delta f)_{z_{k}}+\overline{f_{\bar{z}_{k}}}(\Delta f)_{\bar{z}_{k}}\right]\right\}
\end{aligned}
$$




$$
\begin{aligned}
= & \alpha(\alpha-2)\left(\left\|D_{f}\right\|^{2}+\frac{1}{m}\right)^{\frac{\alpha}{2}-2}\left|\sum_{j=1}^{n} \sum_{k=1}^{n}\left(f_{z_{k} z_{j}} \overline{f_{z_{k}}}+\overline{f_{z_{k} \bar{z}_{j}}} f_{z_{k}}+f_{\bar{z}_{k} z_{j}} \overline{f_{\bar{z}_{k}}}+\overline{f_{\bar{z}_{k} \bar{z}_{j}}} f_{\bar{z}_{k}}\right)\right|^{2} \\
& +2 \alpha\left(\left\|D_{f}\right\|^{2}+\frac{1}{m}\right)^{\frac{\alpha}{2}-1} \sum_{j=1}^{n} \sum_{k=1}^{n}\left(\left|f_{z_{j} z_{k}}\right|^{2}+\left|f_{z_{j} \bar{z}_{k}}\right|^{2}+\left|f_{\bar{z}_{j} z_{k}}\right|^{2}+\left|f_{\bar{z}_{j} \bar{z}_{k}}\right|^{2}\right) \\
& +\alpha\left(\left\|D_{f}\right\|^{2}+\frac{1}{m}\right)^{\frac{\alpha}{2}-1} \operatorname{Re}\left\{\sum_{k=1}^{n}\left[\overline{f_{z_{k}}}(\Delta f)_{z_{k}}+\overline{f_{\bar{z}_{k}}}(\Delta f)_{\bar{z}_{k}}\right]\right\} .
\end{aligned}
$$

By the Cauchy-Schwarz inequality, we have

$$
\begin{aligned}
& \left|\sum_{j=1}^{n} \sum_{k=1}^{n}\left(f_{z_{k} z_{j}} \overline{f_{z_{k}}}+\overline{f_{z_{k} \bar{z}_{j}}} f_{z_{k}}+f_{\bar{z}_{k} z_{j}} \overline{f_{\bar{z}_{k}}}+\overline{f_{\bar{z}_{k} \bar{z}_{j}}} f_{\bar{z}_{k}}\right)\right|^{2} \\
& \leq\left[\sum_{j=1}^{n} \sum_{k=1}^{n}\left(\left|f_{z_{k} z_{j}} \overline{f_{z_{k}}}\right|+\left|\overline{f_{z_{k} \bar{z}_{j}}} f_{z_{k}}\right|+\left|f_{\bar{z}_{k} z_{j}} \overline{f_{\bar{z}_{k}}}\right|+\left|\overline{f_{\bar{z}_{k} \bar{z}_{j}}} f_{\bar{z}_{k}}\right|\right)\right]^{2} \\
& \leq\left\{\sum_{j=1}^{n} \sum_{k=1}^{n}\left[\left(2\left|f_{z_{k}}\right|^{2}+2\left|f_{\bar{z}_{k}}\right|^{2}\right)^{\frac{1}{2}}\left(\left|f_{z_{k} z_{j}}\right|^{2}+\left|f_{z_{k} \bar{z}_{j}}\right|^{2}+\left|f_{\bar{z}_{k} z_{j}}\right|^{2}+\left|f_{\bar{z}_{k} \bar{z}_{j}}\right|^{2}\right)^{\frac{1}{2}}\right]\right\}^{2} \\
& \leq 2 \sum_{j=1}^{n} \sum_{k=1}^{n}\left[\left(2\left|f_{z_{k}}\right|^{2}+2\left|f_{\bar{z}_{k}}\right|^{2}\right)\left(\left|f_{z_{k} z_{j}}\right|^{2}+\left|f_{z_{k} \bar{z}_{j}}\right|^{2}+\left|f_{\bar{z}_{k} z_{j}}\right|^{2}+\left|f_{\bar{z}_{k} \bar{z}_{j}}\right|^{2}\right)\right] \\
& \leq 4\left\|D_{f}\right\|^{2} \sum_{j=1}^{n} \sum_{k=1}^{n}\left(\left|f_{z_{k} z_{j}}\right|^{2}+\left|f_{z_{k} \bar{z}_{j}}\right|^{2}+\left|f_{\bar{z}_{k} z_{j}}\right|^{2}+\left|f_{\bar{z}_{k} \bar{z}_{j}}\right|^{2}\right) .
\end{aligned}
$$

Hence, by (2.7) and Lebesgue's dominated convergence theorem, we obtain

$$
\begin{aligned}
\lim _{m \rightarrow \infty} \Delta\left(F_{m}^{\alpha}\right)= & \alpha(\alpha-2)\left\|D_{f}\right\|^{\alpha-4}\left|\sum_{j=1}^{n} \sum_{k=1}^{n}\left(f_{z_{k} z_{j}} \overline{f_{z_{k}}}+\overline{f_{z_{k} \bar{z}_{j}}} f_{z_{k}}+f_{\bar{z}_{k} z_{j}} \overline{f_{\bar{z}_{k}}}+\overline{f_{\bar{z}_{k} \bar{z}_{j}}} f_{\bar{z}_{k}}\right)\right|^{2} \\
& +2 \alpha\left\|D_{f}\right\|^{\alpha-2} \sum_{j=1}^{n} \sum_{k=1}^{n}\left(\left|f_{z_{j} z_{k}}\right|^{2}+\left|f_{z_{j} \bar{z}_{k}}\right|^{2}+\left|f_{\bar{z}_{j} z_{k}}\right|^{2}+\left|f_{\bar{z}_{j} \bar{z}_{k}}\right|^{2}\right) \\
& +\alpha\left\|D_{f}\right\|^{\alpha-2} \operatorname{Re}\left\{\sum_{k=1}^{n}\left[\overline{f_{z_{k}}}(\Delta f)_{z_{k}}+\overline{f_{\bar{z}_{k}}}(\Delta f)_{\bar{z}_{k}}\right]\right\} \geq 0 .
\end{aligned}
$$

Then, for $\alpha \in[2,4),\left\|D_{f}\right\|^{\alpha}$ is subharmonic in $\mathbf{B}^{n}$.

Proof of Theorem 2 It is not difficult to see that if $\tau$ and $\eta$ are constant functions, then each solution $f$ to (1.2) belongs to $C^{\infty}\left(\mathbf{B}^{n}\right)$, i.e., they are infinitely differentiable in $\mathbf{B}^{n}$.

By elementary calculations, we get

$$
\begin{aligned}
\sum_{k=1}^{n} \operatorname{Re}\left[\overline{f_{z_{k}}}(\Delta f)_{z_{k}}+\overline{f_{\bar{z}_{k}}}(\Delta f)_{\bar{z}_{k}}\right] & =\sum_{k=1}^{n}\left[\tau\left(\left|f_{z_{k}}\right|^{2}+\left|f_{\bar{z}_{k}}\right|^{2}\right)+\frac{\eta}{2}\left|f_{z_{k}}+\overline{f_{\bar{z}_{k}}}\right|^{2}\right] \\
& \leq(\eta+\tau)\left\|D_{f}\right\|^{2}
\end{aligned}
$$

and

$$
\operatorname{Re}(f \overline{\Delta f})=\tau|f|^{2}+\eta(\operatorname{Re}(f))^{2} \leq(\eta+\tau)|f|^{2} .
$$


By using Hölder's inequality, for $\rho \in(0,1)$, we see that

$$
\int_{\partial \mathbf{B}^{n}}\left\|D_{f}(\rho \zeta)\right\|^{p-2}\left(D_{f}^{*}(\rho \zeta)\right)^{2} d \sigma(\zeta) \leq M_{p}^{2}\left(\rho, D_{f}^{*}\right) M_{p}^{p-2}\left(\rho, D_{f}\right)
$$

and

$$
\int_{\partial \mathbf{B}^{n}}\|f(\rho \zeta)\|^{p-2}\left\|D_{f}(\rho \zeta)\right\|^{2} d \sigma(\zeta) \leq M_{p}^{2}\left(\rho, D_{f}\right) M_{p}^{p-2}(\rho, f)
$$

For $t \in[0,1], r \in(0,1)$ and $\rho \in(0, r]$, we obtain

$$
\frac{t\left(1-t^{2 n-2}\right)}{2(n-1)} \leq 1-t
$$

and

$$
\rho \log \frac{r}{\rho} \leq r-\rho
$$

where $n \geq 2$.

Case 1 . Let $n \geq 2$.

Step 1. By (2.7), (2.8), (2.10), Lemma 3, Theorem B and Lebesgue's Dominated Convergence theorem, we see that

$$
\begin{aligned}
M_{p}^{p}\left(r, D_{f}\right)= & \left\|D_{f}(0)\right\|^{p}+\int_{\mathbf{B}^{n}(r)} \Delta\left(\left\|D_{f}(z)\right\|^{p}\right) G_{2 n}(z, r) d V_{N}(z)=\left\|D_{f}(0)\right\|^{p} \\
& +\int_{\mathbf{B}^{n}(r)}\left\{p\left\|D_{f}(z)\right\|^{p-2} \sum_{k=1}^{n} \operatorname{Re} \overline{f_{z_{k}}(z)}(\Delta f(z))_{z_{k}}+\overline{f_{\bar{z}_{k}}(z)}(\Delta f(z))_{\bar{z}_{k}}\right] \\
& +p(p-2)\left\|D_{f}(z)\right\|^{p-4} \mid \sum_{j=1}^{n} \sum_{k=1}^{n}\left(f_{z_{k} z_{j}}(z) \overline{f_{z_{k}}(z)}\right. \\
& \left.+\overline{f_{z_{k} \bar{z}_{j}}(z)} f_{z_{k}}(z)+f_{\bar{z}_{k} z_{j}}(z) \overline{f_{\bar{z}_{k}}(z)}+\overline{f_{\bar{z}_{k} \bar{z}_{j}}(z)} f_{\bar{z}_{k}}(z)\right)\left.\right|^{2} \\
& \left.+2 p\left\|D_{f}(z)\right\|^{p-2}\left(D_{f}^{*}(z)\right)^{2}\right\} G_{2 n}(z, r) d V_{N}(z) \\
\leq & \left\|D_{f}(0)\right\|^{p}+p \int_{\mathbf{B}^{n}(r)}\left[(\eta+\tau)\left\|D_{f}(z)\right\|^{p}\right. \\
& \left.+2(2 p-3)\left\|D_{f}(z)\right\|^{p-2}\left(D_{f}^{*}(z)\right)^{2}\right] G_{2 n}(z, r) d V_{N}(z) \\
= & \left\|D_{f}(0)\right\|^{p}+\frac{p(\eta+\tau)}{2(n-1)} \int_{0}^{r}\left(\rho-\rho^{2 n-1} r^{2(1-n)}\right) M_{p}^{p}\left(\rho, D_{f}\right) d \rho \\
& +\frac{p(2 p-3)}{(n-1)} \int_{0}^{r}\left(\rho-\rho^{2 n-1} r^{2(1-n)}\right) \int_{\partial \mathbf{B}^{n}}\left\|D_{f}(\rho \zeta)\right\|^{p-2}\left(D_{f}^{*}(\rho \zeta)\right)^{2} d \sigma(\zeta) d \rho \\
\leq & \left\|D_{f}(0)\right\|^{p}+\frac{p(\eta+\tau)}{2(n-1)} \int_{0}^{r}\left(\rho-\rho^{2 n-1} r^{2(1-n)}\right) M_{p}^{p}\left(\rho, D_{f}\right) d \rho \\
& +\frac{p(2 p-3)}{(n-1)} \int_{0}^{r}\left(\rho-\rho^{2 n-1} r^{2(1-n)}\right) M_{p}^{2}\left(\rho, D_{f}^{*}\right) M_{p}^{p-2}\left(\rho, D_{f}\right) d \rho . \\
& \\
& \\
&
\end{aligned}
$$


The above together with (2.12) and subharmonicity of $\left\|D_{f}\right\|^{p}$, shows that

$$
\begin{aligned}
& {\left[1-\frac{p(\eta+\tau)}{2(n-1)} \int_{0}^{r}\left(\rho-\rho^{2 n-1} r^{2(1-n)}\right) d \rho\right] M_{p}^{2}\left(r, D_{f}\right)=\left[1-\frac{p r^{2}(\eta+\tau)}{4 n}\right] M_{p}^{2}\left(r, D_{f}\right)} \\
& \leq\left\|D_{f}(0)\right\|^{2}+\frac{p(2 p-3)}{(n-1)} \int_{0}^{r}\left(\rho-\rho^{2 n-1} r^{2(1-n)}\right) M_{p}^{2}\left(\rho, D_{f}^{*}\right) d \rho \\
& =\left\|D_{f}(0)\right\|^{2}+2 p(2 p-3) r^{2} \int_{0}^{1} \frac{t\left(1-t^{2 n-2}\right)}{2(n-1)} M_{p}^{2}\left(r t, D_{f}^{*}\right) d t \\
& \leq\left\|D_{f}(0)\right\|^{2}+2 p(2 p-3) r^{2}\left(M^{*}\right)^{2} \int_{0}^{1}\left[\omega\left(\frac{1}{1-r t}\right)\right]^{2}(1-t) d t \\
& \leq\left\|D_{f}(0)\right\|^{2}+2 p(2 p-3) r^{2}\left(M^{*}\right)^{2} \int_{0}^{1}\left[\omega\left(\frac{1}{1-r t}\right)\right]^{2}(1-r t) d t \\
& \leq\left\|D_{f}(0)\right\|^{2}+2 p(2 p-3) r^{2}\left(M^{*}\right)^{2} \omega(1) \int_{0}^{1} \omega\left(\frac{1}{1-r t}\right) d t .
\end{aligned}
$$

Then

$$
M_{p}^{2}\left(r, D_{f}\right) \leq M_{2}^{*}\left[\left\|D_{f}(0)\right\|^{2}+M_{1}^{*} \int_{0}^{1} \omega\left(\frac{1}{1-r t}\right) d t\right],
$$

where $M_{1}^{*}=2 p(2 p-3)\left(M^{*}\right)^{2} \omega(1)$ and $M_{2}^{*}=1 /[1-p(\eta+\tau) /(4 n)]$.

Step 2. By (2.9), (2.11), Lemmas 1 and 2, we obtain

$$
\begin{aligned}
M_{p}^{p}(r, f)= & |f(0)|^{p}+\int_{\mathbf{B}^{n}(r)} \Delta\left(|f(z)|^{p}\right) G_{2 n}(z, r) d V_{N}(z) \\
\leq & |f(0)|^{p}+\int_{0}^{r} \int_{\partial \mathbf{B}^{n}} 4 n p(p-1) \rho^{2 n-1}|f(\rho \zeta)|^{p-2}\left|D_{f}(\rho \zeta)\right|^{2} G_{2 n}(\rho \zeta, r) d \sigma(\zeta) d \rho \\
& +p(\eta+\tau) \int_{\mathbf{B}^{n}(r)}|f(z)|^{p} G_{2 n}(z, r) d V_{N}(z) \\
\leq & |f(0)|^{p}+4 p(p-1) \int_{0}^{r} n \rho^{2 n-1} G_{2 n}(\rho \zeta, r) M_{p}^{2}\left(\rho, D_{f}\right) M_{p}^{p-2}(\rho, f) d \rho \\
& +\frac{p(\eta+\tau) r^{2}}{4 n} M_{p}^{p}(r, f) .
\end{aligned}
$$

By the above estimates, $(2.12),(2.14)$ and the monotonicity of $M_{p}(r, f)$ on $r$,

$$
\begin{aligned}
\frac{M_{p}^{2}(r, f)}{M_{2}^{*}} \leq & {\left[1-\frac{p(\eta+\tau) r^{2}}{4 n}\right] M_{p}^{2}(r, f) } \\
\leq & |f(0)|^{2}+4 p(p-1) \int_{0}^{r} n \rho^{2 n-1} G_{2 n}(\rho \zeta, r) M_{p}^{2}\left(\rho, D_{f}\right) d \rho \\
= & |f(0)|^{2}+2 p(p-1) \int_{0}^{1} r^{2} M_{p}^{2}\left(r \rho, D_{f}\right) \cdot \frac{\rho\left(1-\rho^{2 n-2}\right)}{2(n-1)} d \rho \\
\leq & |f(0)|^{2}+2 p(p-1) \int_{0}^{1} M_{p}^{2}\left(r \rho, D_{f}\right)(1-\rho) d \rho \\
\leq & |f(0)|^{2}+2 p(p-1) M_{2}^{*}\left\|D_{f}(0)\right\|^{2} \\
& +2 p(p-1) M_{1}^{*} M_{2}^{*} \int_{0}^{1}\left[\int_{0}^{1} \omega\left(\frac{1}{1-r \rho t}\right)(1-\rho) d t\right] d \rho
\end{aligned}
$$




$$
\begin{aligned}
\leq & |f(0)|^{2}+2 p(p-1) M_{2}^{*}\left\|D_{f}(0)\right\|^{2} \\
& +2 p(p-1) M_{1}^{*} M_{2}^{*} \int_{0}^{1}\left[\int_{0}^{1} \omega\left(\frac{1}{1-r \rho t}\right)(1-r t \rho) d t\right] d \rho \\
\leq & |f(0)|^{2}+2 p(p-1) M_{2}^{*}\left\|D_{f}(0)\right\|^{2}+2 p(p-1) M_{1}^{*} M_{2}^{*} \omega(1)<\infty .
\end{aligned}
$$

Hence $f \in \mathcal{H}_{q}^{p}\left(\mathbf{B}^{n}\right)$.

Case 2. Let $n=1$.

Step 3. By (2.7), (2.8), (2.10), Lemma 3, Theorem B and Lebesgue's dominated convergence theorem, we see that

$$
\begin{aligned}
& M_{p}^{p}\left(r, D_{f}\right)=\left\|D_{f}(0)\right\|^{p}+\frac{1}{2} \int_{\mathbf{D}(r)} \Delta\left(\left\|D_{f}(z)\right\|^{p}\right) \log \frac{r}{|z|} d A(z) \\
&=\left\|D_{f}(0)\right\|^{p}+\frac{1}{2} \int_{\mathbf{D}(r)}\left\{p\left\|D_{f}(z)\right\|^{p-2} \operatorname{Re}\left[\overline{f_{z}(z)}(\Delta f(z))_{z}+\overline{f_{\bar{z}}(z)}(\Delta f(z))_{\bar{z}}\right]\right. \\
& \quad+p(p-2)\left\|D_{f}(z)\right\|^{p-4}\left|\left(f_{z z}(z) \overline{f_{z}(z)}+\overline{f_{z \bar{z}}(z)} f_{z}(z)+f_{\bar{z} z}(z) \overline{f_{\bar{z}}(z)}+\overline{f_{\overline{z z}}(z)} f_{\bar{z}}(z)\right)\right|^{2} \\
&\left.\quad+2 p\left\|D_{f}(z)\right\|^{p-2}\left(D_{f}^{*}(z)\right)^{2}\right\} \log \frac{r}{|z|} d A(z) \\
& \leq\left\|D_{f}(0)\right\|^{p}+\frac{p}{2} \int_{\mathbf{D}(r)}\left[(\eta+\tau)\left\|D_{f}(z)\right\|^{p}+2(2 p-3)\left\|D_{f}(z)\right\|^{p-2}\left(D_{f}^{*}(z)\right)^{2}\right] \log \frac{r}{|z|} d A(z) \\
&=\left\|D_{f}(0)\right\|^{p}+p(\eta+\tau) \int_{0}^{r} M_{p}^{p}\left(\rho, D_{f}\right) \rho \log \frac{r}{\rho} d \rho \\
&+2 p(2 p-3) \int_{0}^{r} \rho \log \frac{r}{\rho}\left(\frac{1}{2 \pi} \int_{0}^{2 \pi}\left\|D_{f}\left(\rho e^{i \theta}\right)\right\|^{p-2}\left(D_{f}^{*}\left(\rho e^{i \theta}\right)\right)^{2} d \theta\right) d \rho \\
& \leq\left\|D_{f}(0)\right\|^{p}+p(\eta+\tau) \int_{0}^{r} M_{p}^{p}\left(\rho, D_{f}\right) \rho \log \frac{r}{\rho} d \rho \\
&+2 p(2 p-3) \int_{0}^{r} M_{p}^{2}\left(\rho, D_{f}^{*}\right) M_{p}^{p-2}\left(\rho, D_{f}\right) \rho \log \frac{r}{\rho} d \rho \\
& \leq\left\|D_{f}(0)\right\|^{p}+p(\eta+\tau) M_{p}^{p}\left(r, D_{f}\right) \int_{0}^{r} \rho \log \frac{r}{\rho} d \rho \\
&+2 p(2 p-3) \int_{0}^{r} M_{p}^{2}\left(\rho, D_{f}^{*}\right) M_{p}^{p-2}\left(\rho, D_{f}\right) \rho \log \frac{r}{\rho} d \rho, \\
&+2 p
\end{aligned}
$$

which, together with (2.13), gives that

$$
\begin{aligned}
& {\left[1-p(\eta+\tau) \int_{0}^{r} \rho \log \frac{r}{\rho} d \rho\right] M_{p}^{2}\left(r, D_{f}\right)=\left[1-\frac{p r^{2}(\eta+\tau)}{4}\right] M_{p}^{2}\left(r, D_{f}\right)} \\
& \leq\left\|D_{f}(0)\right\|^{2}+2 p(2 p-3) \int_{0}^{r} \rho \log \frac{r}{\rho} M_{p}^{2}\left(\rho, D_{f}^{*}\right) d \rho \\
& \leq\left\|D_{f}(0)\right\|^{2}+2 p(2 p-3) \int_{0}^{r}(r-\rho) M_{p}^{2}\left(\rho, D_{f}^{*}\right) d \rho \\
& =\left\|D_{f}(0)\right\|^{2}+2 p(2 p-3) r^{2} \int_{0}^{1}(1-t) M_{p}^{2}\left(r t, D_{f}^{*}\right) d t \\
& \leq\left\|D_{f}(0)\right\|^{2}+2 p(2 p-3) r^{2}\left(M^{*}\right)^{2} \int_{0}^{1}\left[\omega\left(\frac{1}{1-r t}\right)\right]^{2}(1-t) d t
\end{aligned}
$$




$$
\begin{aligned}
& \leq\left\|D_{f}(0)\right\|^{2}+2 p(2 p-3) r^{2}\left(M^{*}\right)^{2} \int_{0}^{1}\left[\omega\left(\frac{1}{1-r t}\right)\right]^{2}(1-r t) d t \\
& \leq\left\|D_{f}(0)\right\|^{2}+2 p(2 p-3) r^{2}\left(M^{*}\right)^{2} \omega(1) \int_{0}^{1} \omega\left(\frac{1}{1-r t}\right) d t
\end{aligned}
$$

where $d A$ denotes the normalized area measure in $\mathbf{D}$. Then

$$
M_{p}^{2}\left(r, D_{f}\right) \leq M_{2}^{* *}\left[\left\|D_{f}(0)\right\|^{2}+M_{1}^{*} \int_{0}^{1} \omega\left(\frac{1}{1-r t}\right) d t\right]
$$

where $M_{2}^{* *}=1 /[1-p(\eta+\tau) / 4]$.

Step 4. By (2.9), (2.11), Lemmas 1 and 2, we obtain

$$
\begin{aligned}
M_{p}^{p}(r, f)= & |f(0)|^{p}+\frac{1}{2} \int_{\mathbf{D}(r)} \Delta\left(|f(z)|^{p}\right) \log \frac{r}{|z|} d V_{N}(z) \\
\leq & |f(0)|^{p}+2 p(p-1) \int_{0}^{r}\left(\frac{1}{2 \pi} \int_{0}^{2 \pi}\left|f\left(\rho e^{i \theta}\right)\right|^{p-2}\left|D_{f}\left(\rho e^{i \theta}\right)\right|^{2} d \theta\right) \rho \log \frac{r}{\rho} d \rho \\
& +p(\eta+\tau) \int_{0}^{r}\left(\frac{1}{2 \pi} \int_{0}^{2 \pi}\left|f\left(\rho e^{i \theta}\right)\right|^{p} d \theta\right) \rho \log \frac{r}{\rho} d \rho \\
\leq & |f(0)|^{p}+2 p(p-1) \int_{0}^{r} \rho \log \frac{r}{\rho} M_{p}^{2}\left(\rho, D_{f}\right) M_{p}^{p-2}(\rho, f) d \rho \\
& +p(\eta+\tau) M_{p}^{p}(r, f) \int_{0}^{r} \rho \log \frac{r}{\rho} d \rho \\
\leq & |f(0)|^{p}+2 p(p-1) \int_{0}^{r} \rho \log \frac{r}{\rho} M_{p}^{2}\left(\rho, D_{f}\right) M_{p}^{p-2}(\rho, f) d \rho \\
& +\frac{p r^{2}(\eta+\tau)}{4} M_{p}^{p}(r, f) .
\end{aligned}
$$

The above, (2.13), (2.15) and the monotonicity of $M_{p}(r, f)$ on $r$, imply that

$$
\begin{aligned}
\frac{M_{p}^{2}(r, f)}{M_{2}^{* *} \leq} & {\left[1-\frac{p(\eta+\tau) r^{2}}{4}\right] M_{p}^{2}(r, f) } \\
\leq & |f(0)|^{2}+2 p(p-1) \int_{0}^{r} M_{p}^{2}\left(\rho, D_{f}\right) \rho \log \frac{r}{\rho} d \rho \\
\leq & |f(0)|^{2}+2 p(p-1) \int_{0}^{r} M_{p}^{2}\left(\rho, D_{f}\right)(r-\rho) d \rho \\
\leq & |f(0)|^{2}+2 p(p-1) \int_{0}^{1} M_{p}^{2}\left(r \rho, D_{f}\right)(1-\rho) d \rho \\
\leq & |f(0)|^{2}+2 p(p-1) M_{2}^{* *}\left\|D_{f}(0)\right\|^{2} \\
& +2 p(p-1) M_{1}^{*} M_{2}^{* *} \int_{0}^{1}\left[\int_{0}^{1} \omega\left(\frac{1}{1-r \rho t}\right)(1-\rho) d t\right] d \rho \\
\leq & |f(0)|^{2}+2 p(p-1) M_{2}^{* *}\left\|D_{f}(0)\right\|^{2} \\
& +2 p(p-1) M_{1}^{*} M_{2}^{* *} \int_{0}^{1}\left[\int_{0}^{1} \omega\left(\frac{1}{1-r \rho t}\right)(1-r t \rho) d t\right] d \rho \\
\leq & |f(0)|^{2}+2 p(p-1) M_{2}^{* *}\left\|D_{f}(0)\right\|^{2}+2 p(p-1) M_{1}^{*} M_{2}^{* *} \omega(1)<\infty .
\end{aligned}
$$

Hence $f \in \mathcal{H}_{g}^{p}(\mathbf{D})$. The proof of the theorem is complete. 


\section{Dirichlet-type spaces, Bergman-type spaces and applications to PDEs}

Proof of Theorem 3. We first prove the necessity. Since $\operatorname{Re}(f \overline{\Delta f}) \geq 0$, we observe that $\Delta\left(|f|^{p}\right) \geq 0$ and

$$
0 \leq \int_{\mathbf{B}^{n}}\left(1-|z|^{2}\right)^{\alpha} \Delta\left(|f(z)|^{p}\right) d V_{N}(z)<\infty .
$$

Let $r \in(0,1)$. For $\alpha \geq 2$, it is not difficult to see that

$$
\left.\left(r^{2}-|z|^{2}\right)^{\alpha}\right|_{\partial \mathbf{B}^{n}(r)}=0 \quad \text { and }\left.\quad \frac{\partial}{\partial \varepsilon}\left[\left(r^{2}-|z|^{2}\right)^{\alpha}\right]\right|_{\partial \mathbf{B}^{n}(r)}=0
$$

where $\partial / \partial \varepsilon$ denotes an outer normal derivative. Then, by Green's theorem, we get

$$
\begin{aligned}
& \int_{\mathbf{B}^{n}(r)}\left(r^{2}-|z|^{2}\right)^{\alpha} \Delta\left(|f(z)|^{p}\right) d V_{N}(z)=\int_{\mathbf{B}^{n}(r)}|f(z)|^{p} \Delta\left[\left(r^{2}-|z|^{2}\right)^{\alpha}\right] d V_{N}(z) \\
& =4 \alpha \int_{\mathbf{B}^{n}(r)}|f(z)|^{p}\left(r^{2}-|z|^{2}\right)^{\alpha-2}\left[|z|^{2}(n+\alpha-1)-n r^{2}\right] d V_{N}(z),
\end{aligned}
$$

which, together with (3.1), gives that

$$
\begin{aligned}
\infty> & 4 \alpha \int_{\mathbf{B}^{n}\left(R_{1}\right)}|f(z)|^{p}\left(1-|z|^{2}\right)^{\alpha-2}\left[n-|z|^{2}(n+\alpha-1)\right] d V_{N}(z) \\
& +\int_{\mathbf{B}^{n}}\left(1-|z|^{2}\right)^{\alpha} \Delta\left(|f(z)|^{p}\right) d V_{N}(z) \\
\geq & 4 \alpha \int_{\mathbf{B}^{n}\left(r R_{1}\right)}|f(z)|^{p}\left(1-|z|^{2}\right)^{\alpha-2}\left[n-|z|^{2}(n+\alpha-1)\right] d V_{N}(z) \\
& +\int_{\mathbf{B}^{n}(r)}\left(1-|z|^{2}\right)^{\alpha} \Delta\left(|f(z)|^{p}\right) d V_{N}(z) \\
\geq & 4 \alpha \int_{\mathbf{B}^{n}\left(r R_{1}\right)}|f(z)|^{p}\left(r^{2}-|z|^{2}\right)^{\alpha-2}\left[n r^{2}-|z|^{2}(n+\alpha-1)\right] d V_{N}(z) \\
& +\int_{\mathbf{B}^{n}(r)}\left(r^{2}-|z|^{2}\right)^{\alpha} \Delta\left(|f(z)|^{p}\right) d V_{N}(z) \\
= & 4 \alpha \int_{\mathbf{B}^{n}(r) \backslash \mathbf{B}^{n}\left(r R_{1}\right)}|f(z)|^{p}\left(r^{2}-|z|^{2}\right)^{\alpha-2}\left[|z|^{2}(n+\alpha-1)-n r^{2}\right] d V_{N}(z) \\
\geq & 2 r^{2} \alpha \int_{\mathbf{B}^{n}(r) \backslash \mathbf{B}^{n}\left(r R_{2}\right)}|f(z)|^{p}\left(r^{2}-|z|^{2}\right)^{\alpha-2} d V_{N}(z),
\end{aligned}
$$

where $R_{1}=\sqrt{\frac{n}{n+\alpha-1}}$ and $R_{2}=\sqrt{\frac{n+\frac{1}{2}}{n+\alpha-1}}$.

For $R_{2}<r<1$, we conclude that

$$
\infty>2 r^{2} \alpha \int_{\mathbf{B}^{n}(r) \backslash \mathbf{B}^{n}\left(r R_{2}\right)}|f(z)|^{p}\left(r^{2}-|z|^{2}\right)^{\alpha-2} d V_{N}(z) \geq 2 \alpha R_{2}^{2} U(r),
$$

where

$$
U(r)=\int_{\mathbf{B}^{n}(r) \backslash \mathbf{B}^{n}\left(R_{2}\right)}|f(z)|^{p}\left(r^{2}-|z|^{2}\right)^{\alpha-2} d V_{N}(z) .
$$

Then, for $R_{2}<r<1, U(r)$ is increasing and bounded, from which we conclude that

$$
\lim _{r \rightarrow 1-} U(r)
$$

exists. Hence for $p \geq 2, f \in b_{\alpha-2, p}\left(\mathbf{B}^{n}\right)$. 
Next we prove the sufficiency. For $\alpha \geq 2$, by (3.2), we have

$$
\begin{aligned}
& 4 \alpha \int_{\mathbf{B}^{n}\left(r R_{1}\right)}|f(z)|^{p}\left(r^{2}-|z|^{2}\right)^{\alpha-2}\left[n r^{2}-|z|^{2}(n+\alpha-1)\right] d V_{N}(z) \\
& \quad+\int_{\mathbf{B}^{n}(r)}\left(r^{2}-|z|^{2}\right)^{\alpha} \Delta\left(|f(z)|^{p}\right) d V_{N}(z) \\
& =4 \alpha \int_{\mathbf{B}^{n}(r) \backslash \mathbf{B}^{n}\left(r R_{1}\right)}|f(z)|^{p}\left(r^{2}-|z|^{2}\right)^{\alpha-2}\left[|z|^{2}(n+\alpha-1)-n r^{2}\right] d V_{N}(z) \\
& \leq 4 \alpha(n+\alpha-1) \int_{\mathbf{B}^{n}(r) \backslash \mathbf{B}^{n}\left(r R_{1}\right)}|f(z)|^{p}\left(r^{2}-|z|^{2}\right)^{\alpha-2} d V_{N}(z) \\
& \leq 4 \alpha(n+\alpha-1) \int_{\mathbf{B}^{n}}|f(z)|^{p}\left(1-|z|^{2}\right)^{\alpha-2} d V_{N}(z)<\infty .
\end{aligned}
$$

Since

$$
\begin{aligned}
\infty & >\int_{\mathbf{B}^{n}\left(R_{1}\right)}|f(z)|^{p}\left(1-|z|^{2}\right)^{\alpha-2}\left[n-|z|^{2}(n+\alpha-1)\right] d V_{N}(z) \\
& \geq \int_{\mathbf{B}^{n}\left(r R_{1}\right)}|f(z)|^{p}\left(r^{2}-|z|^{2}\right)^{\alpha-2}\left[n r^{2}-|z|^{2}(n+\alpha-1)\right] d V_{N}(z),
\end{aligned}
$$

which, together with (3.4) and $\Delta\left(|f|^{p}\right) \geq 0$, implies that

$$
\lim _{r \rightarrow 1-} \int_{\mathbf{B}^{n}(r)}\left(r^{2}-|z|^{2}\right)^{\alpha} \Delta\left(|f(z)|^{p}\right) d V_{N}(z)
$$

does exist. Therefore,

$$
\int_{\mathbf{B}^{n}}\left(1-|z|^{2}\right)^{\alpha} \Delta\left(|f(z)|^{p}\right) d V_{N}(z)<\infty
$$

and thus the theorem is proved.

The following result is well-known.

Lemma 4. Suppose that $a, b \in[0, \infty)$ and $q \in(0, \infty)$. Then

$$
(a+b)^{q} \leq 2^{\max \{q-1,0\}}\left(a^{q}+b^{q}\right) .
$$

Proof of Theorem 4. By Lemma 3, for $\rho \in[0, d(z))$, we get

$$
\left\|D_{f}(z)\right\|^{\alpha} \leq \int_{\partial \mathbf{B}^{n}}\left\|D_{f}(z+\rho \zeta)\right\|^{\alpha} d \sigma(\zeta) .
$$

Multiplying both sides of the inequality (3.6) by $2 n \rho^{2 n-1}$ and integrating from 0 to $d(z) / 2$, we have

$$
\begin{aligned}
\frac{d(z)^{2 n}\left\|D_{f}(z)\right\|^{\alpha}}{2^{2 n}} & \leq \int_{\partial \mathbf{B}^{n}} \int_{0}^{\frac{d(z)}{2}} 2 n \rho^{2 n-1}\left\|D_{f}(z+\rho \zeta)\right\|^{\alpha} d \rho d \sigma(\zeta) \\
& =\int_{\mathbf{B}^{n}\left(z, \frac{d(z)}{2}\right)}\left\|D_{f}(\xi)\right\|^{\alpha} d V_{N}(\xi) \\
& \leq 2^{\gamma}(d(z))^{-\gamma} \int_{\mathbf{B}^{n}\left(z, \frac{d(z)}{2}\right)}(1-|\xi|)^{\gamma}\left\|D_{f}(\xi)\right\|^{\alpha} d V_{N}(\xi) \\
& \leq \frac{2^{\gamma}\|f\|_{\mathcal{D}_{\gamma, \alpha}}^{\alpha}}{(d(z))^{\gamma}}
\end{aligned}
$$


which implies that

$$
\left\|D_{f}(z)\right\| \leq \frac{M_{1}}{(d(z))^{q+1}}
$$

where $M_{1}=2^{1+q}\|f\|_{\mathcal{D}_{\gamma, \alpha}}$ and $q=\frac{\gamma+2 n}{\alpha}-1$. By (3.7), we know that

$$
\begin{aligned}
|f(z)| & \leq|f(0)|+\left|\int_{[0, z]} d f(\varsigma)\right| \leq|f(0)|+\sqrt{2} \int_{[0, z]}\left\|D_{f}(\varsigma)\right\|\|d \varsigma\| \\
& \leq|f(0)|+\frac{M_{2}}{(d(z))^{q}}
\end{aligned}
$$

where $M_{2}=M_{1} \sqrt{2} / q$ and $[0, z]$ denotes the line segment from 0 to $z$.

By (3.8) and Lemma 4, we see that for $z \in \mathbf{B}^{n}$,

$$
\begin{aligned}
& |f(z)|^{p-2} \leq\left[|f(0)|+\frac{M_{2}}{(d(z))^{q}}\right]^{p-2} \leq 2^{p-2}\left[|f(0)|^{p-2}+\frac{M_{2}^{p-2}}{(d(z))^{q(p-2)}}\right] \\
& |f(z)|^{p-1} \leq\left[|f(0)|+\frac{M_{2}}{(d(z))^{q}}\right]^{p-1} \leq 2^{p-1}\left[|f(0)|^{p-1}+\frac{M_{2}^{p-1}}{(d(z))^{q(p-1)}}\right]
\end{aligned}
$$

and

$$
|f(z)|^{p} \leq\left[|f(0)|+\frac{M_{2}}{(d(z))^{q}}\right]^{p} \leq 2^{p}\left[|f(0)|^{p}+\frac{M_{2}^{p}}{(d(z))^{q p}}\right] .
$$

Case 1. Let $p \in[4, \infty)$. By direct calculations, we get

$$
\begin{aligned}
\Delta\left(|f|^{p}\right) & =p(p-2)|f|^{p-4} \sum_{k=1}^{n}\left|f_{z_{k}} \bar{f}+\overline{f_{\bar{z}}} f\right|^{2}+2 p|f|^{p-2}\left\|D_{f}\right\|^{2}+p|f|^{p-2} \operatorname{Re}(f \overline{\Delta f}) \\
& \leq p(p-2)|f|^{p-4} \sum_{k=1}^{n}\left|f_{z_{k}} \bar{f}+\overline{f_{\overline{z_{k}}}} f\right|^{2}+2 p|f|^{p-2}\left\|D_{f}\right\|^{2}+p|f|^{p-1}|\Delta f| \\
& \leq 2 p(p-1)|f|^{p-2}\left\|D_{f}\right\|^{2}+p a|f|^{p-1}\left\|D_{f}\right\|+p b|f|^{p}+p c|f|^{p-1} .
\end{aligned}
$$

It follows from (3.9), (3.10), (3.11) and (3.12) that

$$
\begin{aligned}
(d(z))^{p q} \Delta\left(|f|^{p}\right) \leq & 2 p(p-1)(d(z))^{p q}|f|^{p-2}\left\|D_{f}\right\|^{2} \\
& +p a(d(z))^{p q}|f|^{p-1}\left\|D_{f}\right\|+p b(d(z))^{p q}|f|^{p} \\
& +p c(d(z))^{p q}|f|^{p-1} \\
= & 2 p(p-1)(d(z))^{p q-\frac{2 \gamma}{\alpha}}|f|^{p-2}\left\|D_{f}\right\|^{2}(d(z))^{\frac{2 \gamma}{\alpha}} \\
& +p a(d(z))^{p q-\frac{\gamma}{\alpha}}|f|^{p-1}\left\|D_{f}\right\|(d(z))^{\frac{\gamma}{\alpha}} \\
& +p b(d(z))^{p q}|f|^{p}+p c(d(z))^{p q}|f|^{p-1} \\
\leq & M_{3}\left\|D_{f}\right\|^{2}(d(z))^{\frac{2 \gamma}{\alpha}}+M_{4}\left\|D_{f}\right\|(d(z))^{\frac{\gamma}{\alpha}}+M_{5}
\end{aligned}
$$

where

$$
\begin{aligned}
& M_{3}=2^{p-1} p(p-1)\left(|f(0)|^{p-2}+M_{2}^{p-2}\right) \\
& M_{4}=p 2^{p-1}\left(|f(0)|^{p-1}+M_{2}^{p-1}\right) \sup _{z \in \mathbf{B}^{n}} a(z)
\end{aligned}
$$


and

$$
M_{5}=p 2^{p}\left(|f(0)|^{p}+M_{2}^{p}\right) \sup _{z \in \mathbf{B}^{n}} b(z)+p M 2^{p-1}\left(|f(0)|^{p-1}+M_{2}^{p-1}\right) .
$$

By Hölder's inequality, we obtain

$$
\begin{aligned}
& \int_{\mathbf{B}^{n}}(d(z))^{\frac{2 \gamma}{\alpha}}\left\|D_{f}(z)\right\|^{2} d V_{N}(z) \\
& \leq\left(\int_{\mathbf{B}^{n}}(d(z))^{\gamma}\left\|D_{f}(z)\right\|^{\alpha} d V_{N}(z)\right)^{\frac{2}{\alpha}}\left(\int_{\mathbf{B}^{n}} d V_{N}(z)\right)^{1-\frac{2}{\alpha}} \leq\|f\|_{\mathcal{D}_{\gamma, \alpha}}^{2}
\end{aligned}
$$

which gives

$$
\begin{aligned}
& \int_{\mathbf{B}^{n}}(d(z))^{\frac{\gamma}{\alpha}}\left\|D_{f}(z)\right\| d V_{N}(z) \\
& \leq\left(\int_{\mathbf{B}^{n}}(d(z))^{\frac{2 \gamma}{\alpha}}\left\|D_{f}(z)\right\|^{2} d V_{N}(z)\right)^{\frac{1}{2}}\left(\int_{\mathbf{B}^{n}} d V_{N}(z)\right)^{\frac{1}{2}} \leq\|f\|_{\mathcal{D}_{\gamma, \alpha}} .
\end{aligned}
$$

It follows from (3.13), (3.14) and (3.15) that

$$
\begin{aligned}
& \int_{\mathbf{B}^{n}}(d(z))^{p q} \Delta\left(|f(z)|^{p}\right) d V_{N}(z) \\
& =\int_{\mathbf{B}^{n}}\left[M_{3}\left\|D_{f}\right\|^{2}(d(z))^{\frac{2 \gamma}{\alpha}}+M_{4}\left\|D_{f}\right\|(d(z))^{\frac{\gamma}{\alpha}}+M_{5}\right] d V_{N}(z) \\
& \leq M_{3}\|f\|_{\mathcal{D}_{\gamma, \alpha}}^{2}+M_{4}\|f\|_{\mathcal{D}_{\gamma, \alpha}}+M_{5}<\infty
\end{aligned}
$$

Case 2. Let $p \in[2,4)$. For $p \in[2,4), m \in\{1,2, \ldots\}$ and $r \in(0,1)$, let $T_{m}^{p}=$ $\left(|f|^{2}+\frac{1}{m}\right)^{\frac{p}{2}}$. Then, by (3.13), (3.14), (3.15) and Lebesgue's dominated convergence theorem, we have

$$
\begin{aligned}
\lim _{r \rightarrow 1-}\left\{\lim _{m \rightarrow \infty} \int_{\mathbf{B}^{n}(r)}(d(z))^{p q} \Delta\left(T_{m}^{p}(z)\right) d V_{N}(z)\right\} \\
=\lim _{r \rightarrow 1-} \int_{\mathbf{B}^{n}(r)}(d(z))^{p q} \lim _{m \rightarrow \infty} \Delta\left(T_{m}^{p}(z)\right) d V_{N}(z) \\
=\lim _{r \rightarrow 1-} \int_{\mathbf{B}^{n}(r)}(d(z))^{p q}\left[p(p-2)|f(z)|^{p-4} \sum_{k=1}^{n} \mid f_{z_{k}}(z) \overline{f(z)}\right. \\
\left.+\left.\overline{f_{\bar{z}_{k}}(z)} f(z)\right|^{2}+2 p|f(z)|^{p-2}\left\|D_{f}(z)\right\|^{2}+p|f(z)|^{p-2} \operatorname{Re}(f(z) \overline{\Delta f(z)})\right] d V_{N}(z) \\
\leq \int_{\mathbf{B}^{n}}\left(M_{3}\left\|D_{f}\right\|^{2}(d(z))^{\frac{2 \gamma}{\alpha}}+M_{4}\left\|D_{f}\right\|(d(z))^{\frac{\gamma}{\alpha}}+M_{5}\right) d V_{N}(z)<\infty .
\end{aligned}
$$

This concludes the proof of the theorem.

Proof of Theorem 5. Case 1. Let $n \geq 2$. Without loss of generality, we may assume that

$$
\inf _{z \in \mathbf{B}^{n}} a_{1}(z)>0 \text { and } \inf _{z \in \mathbf{B}^{n}} b_{1}(z)>0
$$


Let $r_{0} \in(0,1)$ be a constant. Then, by Lemma 2 , for $0<r_{0} \leq r<1$, we have

$$
\begin{aligned}
& M_{p}^{p}(r, f)=|f(0)|^{p}+\int_{\mathbf{B}^{n}(r)} \Delta\left(|f(z)|^{p}\right) G_{2 n}(z, r) d V_{N}(z) \\
& \geq|f(0)|^{p}+\int_{\mathbf{B}^{n}(r)}\left(a_{1}(z)\left\|D_{f}(z)\right\|^{t_{1}}+b_{1}(z)|f(z)|^{t_{2}}+c_{1}(z)\right) G_{2 n}(z, r) d V_{N}(z) \\
& \geq|f(0)|^{p}+\inf _{z \in \mathbf{B}^{n}} a_{1}(z) \int_{\mathbf{B}^{n}(r)}\left\|D_{f}(z)\right\|^{t_{1}} G_{2 n}(z, r) d V_{N}(z) \\
& \quad+\inf _{z \in \mathbf{B}^{n}} b_{1}(z) \int_{\mathbf{B}^{n}(r)}|f(z)|^{t_{2}} G_{2 n}(z, r) d V_{N}(z) \\
& \quad+\inf _{z \in \mathbf{B}^{n}} c_{1}(z) \int_{\mathbf{B}^{n}(r)} G_{2 n}(z, r) d V_{N}(z) \\
& =|f(0)|^{p}+\inf _{z \in \mathbf{B}^{n}} a_{1}(z) \int_{\mathbf{B}^{n}\left(r_{0}\right)}\left\|D_{f}(z)\right\|^{t_{1}} G_{2 n}(z, r) d V_{N}(z) \\
& \quad+\inf _{z \in \mathbf{B}^{n}} a_{1}(z) \int_{\mathbf{B}^{n}(r) \backslash \mathbf{B}^{n}\left(r_{0}\right)}\left\|D_{f}(z)\right\|^{t_{1}} G_{2 n}(z, r) d V_{N}(z) \\
& \quad+\inf _{z \in \mathbf{B}^{n}} b_{1}(z) \int_{\mathbf{B}^{n}\left(r_{0}\right)}|f(z)|^{t_{2}} G_{2 n}(z, r) d V_{N}(z) \\
& \quad+\inf _{z \in \mathbf{B}^{n}} b_{1}(z) \int_{\mathbf{B}^{n}(r) \backslash \mathbf{B}^{n}\left(r_{0}\right)}|f(z)|^{t_{2}} G_{2 n}(z, r) d V_{N}(z) \\
& \quad+\inf _{z \in \mathbf{B}^{n}} c_{1}(z) \int_{\mathbf{B}^{n}(r)} G_{2 n}(z, r) d V_{N}(z) .
\end{aligned}
$$

It is easy to see that, for all $r \in(0,1)$,

$$
0<\int_{\mathbf{B}^{n}(r)} G_{2 n}(z, r) d V_{N}(z)<\infty .
$$

Since

$$
\int_{\mathbf{B}^{n}\left(r_{0}\right)}\left\|D_{f}(z)\right\|^{t_{1}} G_{2 n}(z, r) d V_{N}(z) \leq \int_{\mathbf{B}^{n}\left(r_{0}\right)}\left\|D_{f}(z)\right\|^{t_{1}} G_{2 n}(z, 1) d V_{N}(z)<\infty
$$

and

$$
\begin{aligned}
\infty & \left.>\int_{\mathbf{B}^{n}(r) \backslash \mathbf{B}^{n}\left(r_{0}\right)}\left\|D_{f}(z)\right\|^{t_{1}} G_{2 n}(z, r)\right) d V_{N}(z) \\
& =\frac{1}{4 n(n-1)} \int_{\mathbf{B}^{n}(r) \backslash \mathbf{B}^{n}\left(r_{0}\right)} \frac{(r-|z|)\left(\sum_{k=0}^{2 n-3} r^{2 n-3-k}|z|^{k}\right)}{|z|^{2 n-2} r^{2 n-2}}\left\|D_{f}(z)\right\|^{t_{1}} d V_{N}(z) \\
& \geq \delta(r),
\end{aligned}
$$

which, together with $f \in \mathcal{H}_{g}^{p}\left(\mathbf{B}^{n}\right),(3.16),(3.17)$ and the monotonicity of $\delta(r)$, yield that the limit

$$
\lim _{r \rightarrow 1-} \int_{\mathbf{B}^{n}(r) \backslash \mathbf{B}^{n}\left(r_{0}\right)}(r-|z|)\left\|D_{f}(z)\right\|^{t_{1}} d V_{N}(z)
$$

exists, where

$$
\delta(r)=\frac{r_{0}^{2 n-3}}{2 n} \int_{\mathbf{B}^{n}(r) \backslash \mathbf{B}^{n}\left(r_{0}\right)}(r-|z|)\left\|D_{f}(z)\right\|^{t_{1}} d V_{N}(z) .
$$


Then $f \in \mathcal{D}_{1, t_{1}}\left(\mathbf{B}^{n}\right)$.

By using a similar argument as in the proof of (3.18), we see that $f \in b_{1, t_{2}}\left(\mathbf{B}^{n}\right)$.

Case 2. Let $n=1$. In this case, the proof is similar to the proof of the case 2 in Theorem 1. Therefore, proof of the theorem is complete.

Proof of Corollary 1.6. Without loss of generality, we assume that $\prod_{k=1}^{n} \lambda_{k} \neq 0$. Case 1. Let $p \in[4, \infty)$. By computations, for $k \in\{1, \ldots, n\}$, we have

$$
\begin{aligned}
& \left(|f|^{p}\right)_{z_{k} \bar{z}_{k}}=\left[\left(f^{\frac{p}{2}} f^{\frac{p}{2}}\right)_{\bar{z}_{k}}\right]_{z_{k}}=\frac{p}{2}\left(f^{\frac{p}{2}-1} f_{\bar{z}_{k}} f^{\frac{p}{2}}+f^{\frac{p}{2}} f^{\frac{p}{2}-1} \overline{f_{z_{k}}}\right)_{z_{k}} \\
& =\frac{p}{2}\left(\lambda_{k} f^{\frac{p}{2}+\frac{\alpha}{2}-1} \bar{f}^{\frac{p}{2}+\frac{\alpha}{2}}+f^{\frac{p}{2}} f^{\frac{p}{2}-1} \overline{f_{z_{k}}}\right)_{z_{k}} \\
& =\frac{p}{2}\left[\lambda_{k}\left(\frac{p}{2}+\frac{\alpha}{2}-1\right) f^{\frac{p}{2}+\frac{\alpha}{2}-2} \bar{f}^{\frac{p}{2}+\frac{\alpha}{2}} f_{z_{k}}+\lambda_{k}\left(\frac{p}{2}+\frac{\alpha}{2}\right)|f|^{p+\alpha-2} \overline{f_{\bar{z}_{k}}}\right. \\
& \left.+\frac{p}{2}|f|^{p-2}\left|f_{z_{k}}\right|^{2}+\left(\frac{p}{2}-1\right) \overline{f_{z_{k}}} f^{\frac{p}{2}} f^{\frac{p}{2}-2} \overline{f_{\bar{z}_{k}}}+f^{\frac{p}{2}} f^{\frac{p}{2}-1} \overline{f_{z_{k} \bar{z}_{k}}}\right] \\
& =\frac{p}{2}\left[\lambda_{k}\left(\frac{p}{2}+\frac{\alpha}{2}-1\right)|f|^{p+\alpha-4} f_{z_{k}} \bar{f}^{2}+\lambda_{k}^{2}\left(\frac{p+\alpha}{2}\right)|f|^{p+\alpha-2}|f|^{\alpha}\right. \\
& \left.+\frac{p}{2}|f|^{p-2}\left|f_{z_{k}}\right|^{2}+\lambda_{k}\left(\frac{\alpha}{2}+\frac{p}{2}-1\right)|f|^{p+\alpha-4} \overline{f_{z_{k}}} f^{2}+\frac{\alpha \lambda_{k}^{2}}{2}|f|^{p+2 \alpha-2}\right] \\
& =\frac{p}{2}\left[\lambda_{k}\left(\frac{p}{2}+\frac{\alpha}{2}-1\right)|f|^{p+\alpha-4} f_{z_{k}} \bar{f}^{2}+\lambda_{k}\left(\frac{p}{2}+\frac{\alpha}{2}-1\right)|f|^{p+\alpha-4} \overline{f_{z_{k}}} f^{2}\right. \\
& \left.+\lambda_{k}^{2}\left(\alpha+\frac{p}{2}\right)|f|^{p+2 \alpha-2}+\frac{p}{2}\left|f_{z_{k}}\right|^{2}|f|^{p-2}\right] \\
& =\frac{p}{2}\left\{\operatorname{Re}\left[\lambda_{k}(p+\alpha-2)|f|^{p+\alpha-4} f_{z_{k}} \bar{f}^{2}\right]+\lambda_{k}^{2}\left(\alpha+\frac{p}{2}\right)|f|^{p+2 \alpha-2}\right. \\
& \left.+\frac{p}{2}\left|f_{z_{k}}\right|^{2}|f|^{p-2}\right\} \text {, }
\end{aligned}
$$

which implies

$$
\begin{aligned}
\Delta\left(|f|^{p}\right)=4 \sum_{k=1}^{n}\left(|f|^{p}\right)_{z_{k} \bar{z}_{k}}= & 2 p\left\{\sum_{k=1}^{n} \operatorname{Re}\left[\lambda_{k}(p+\alpha-2)|f|^{p+\alpha-4} f_{z_{k}} \bar{f}^{2}\right]\right. \\
& \left.+\sum_{k=1}^{n} \lambda_{k}^{2}\left(\alpha+\frac{p}{2}\right)|f|^{p+2 \alpha-2}+\frac{p}{2} \sum_{k=1}^{n}\left|f_{z_{k}}\right|^{2}|f|^{p-2}\right\} .
\end{aligned}
$$

Hence,

$$
\begin{aligned}
& \Delta\left(|f|^{p}\right)-\left[4 p-(2-\alpha)^{2}\right]|f|^{p+2 \alpha-2} \sum_{k=1}^{n} \lambda_{k}^{2} \\
& =\sum_{k=1}^{n}\left\{\lambda_{k}^{2}\left[p^{2}+2 p(\alpha-2)+(\alpha-2)^{2}\right]|f|^{p+2 \alpha-2}\right. \\
& \left.\quad+\operatorname{Re}\left[2 p \lambda_{k}(p+\alpha-2)|f|^{p+\alpha-4} f_{z_{k}} \bar{f}^{2}\right]+p^{2}\left|f_{z_{k}}\right|^{2}|f|^{p-2}\right\} \\
& \geq \sum_{k=1}^{n}\left\{\lambda_{k}^{2}(p+\alpha-2)^{2}|f|^{p+2 \alpha-2}+p^{2}\left|f_{z_{k}}\right|^{2}|f|^{p-2}\right. \\
& \left.\quad-\left|2 p \lambda_{k}(p+\alpha-2)\right||f|^{p+\alpha-2}\left|f_{z_{k}}\right|\right\} \\
& =|f|^{p-2} \sum_{k=1}^{n}\left(\left|\lambda_{k}(p+\alpha-2)\right||f|^{\alpha}-|p|\left|f_{z_{k}}\right|\right)^{2} \geq 0,
\end{aligned}
$$


which yields

$$
\Delta\left(|f|^{p}\right) \geq\left[4 p-(2-\alpha)^{2}\right]|f|^{p+2 \alpha-2} \sum_{k=1}^{n} \lambda_{k}^{2} .
$$

Case 2. Let $p \in[2,4)$. For $m \in\{1,2, \ldots\}$, let $G_{m}^{p}=\left(|f|^{2}+\frac{1}{m}\right)^{\frac{p}{2}}$. Then by Lebesgue's dominated convergence theorem and (3.19), we have

$$
\begin{aligned}
\lim _{m \rightarrow \infty} \Delta\left(G_{m}^{p}\right)= & \lim _{m \rightarrow \infty} 4 \sum_{k=1}^{n}\left(G_{m}^{p}\right)_{z_{k} \bar{z}_{k}} \\
= & 2 p\left\{\sum_{k=1}^{n} \operatorname{Re}\left[\lambda_{k}(p+\alpha-2)|f|^{p+\alpha-4} f_{z_{k}} \bar{f}^{2}\right]\right. \\
& \left.+\sum_{k=1}^{n} \lambda_{k}^{2}\left(\alpha+\frac{p}{2}\right)|f|^{p+2 \alpha-2}+\frac{p}{2} \sum_{k=1}^{n}\left|f_{z_{k}}\right|^{2}|f|^{p-2}\right\} \\
\geq & {\left[4 p-(2-\alpha)^{2}\right]|f|^{p+2 \alpha-2} \sum_{k=1}^{n} \lambda_{k}^{2} . }
\end{aligned}
$$

Applying Theorem 5, we conclude that $f \in b_{1, \vartheta}\left(\mathbf{B}^{n}\right)$, where $\vartheta=p+2 \alpha-2$. The proof of the Corollary is complete.

Acknowledgements. We thank the referee for providing constructive comments and help in improving this paper. This research was partly supported by the National Natural Science Foundation of China (No. 11571216 and No. 11401184), the Science and Technology Plan Project of Hunan Province (No. 2016TP1020), the Science and Technology Plan Project of Hengyang City (2017KJ183), and Hunan Province double First-class Construction Application characteristic discipline Project (Xiangjiaotong [2018] 469).

\section{References}

[1] Arfken, G.: Mathematical methods for physicists. - Academic Press, Orlando, FL, 3rd ed., 1985.

[2] Astala, K., T. Inaniec, and G. Martin: Elliptic partial differential equations and quasiconformal mappings in the plane. - Princeton Math. Ser. 48, Princeton Univ. Press, Princeton, 2009.

[3] Chen, S. L., S. Ponnusamy, and A. Rasila: On characterizations of Bloch-type, Hardy-type and Lipschitz-type spaces. - Math. Z. 279, 2015, 163-183.

[4] Chen, S. L., S. Ponnusamy, and X. Wang: Integral means and coefficient estimates on planar harmonic mappings. - Ann. Acad. Sci. Fenn. Math. 37, 2012, 69-79.

[5] Chen, S. L., S. Ponnusamy, and X. Wang: Weighted Lipschitz continuity, Schwarz-Pick's Lemma and Landau-Bloch's theorem for hyperbolic harmonic functions in the unit ball. Math. Model. Anal. 18, 2013, 66-79.

[6] Chen, S. L., and A. Rasila: Schwarz-Pick type estimates of pluriharmonic mappings in the unit polydisk. - Illinois J. Math. 58, 2014, 1015-1024.

[7] Chen, S. L., A. Rasila, and X. Wang: Radial growth, Lipschitz and Dirichlet spaces on solutions to the non-homogenous Yukawa equation. - Israel J. Math. 204, 2014, 261-282.

[8] Coffman, A., and Y. Pan: Some nonlinear differential inequalities and an application to Hölder continuous almost complex structures. - Ann. Inst. H. Poincaré Anal. Non Linéaire 28, 2011, 149-157.

[9] Dоов, J. L.: Classical potential theory and its probabilistic counterpart. - Springer, New York, 1984. 
[10] Duffin, R. J.: Yukawa potential theory. - J. Math. Anal. Appl. 35, 1971, 104-130.

[11] Duffin, R. J.: Hilbert transforms in Yukawan potential theory. - Proc. Nat. Acad. Sci. U.S.A. 69, 1972, 3677-3679.

[12] Duren, P.: Theory of $H^{p}$ spaces. - Dover, Mineola, N.Y., 2nd ed., 2000.

[13] Duren, P., and A. P. Schuster: Bergman spaces. - Amer. Math. Soc., Providence, 2004.

[14] Dyakonov, K. M.: Equivalent norms on Lipschitz-type spaces of holomorphic functions. Acta Math. 178, 1997, 143-167.

[15] Dyakonov, K. M.: Holomorphic functions and quasiconformal mappings with smooth moduli. - Adv. Math. 187, 2004, 146-172.

[16] Evans, L. C.: Partial differential equations. - Amer. Math. Soc., 1998.

[17] Girela, D., M. Pavlović, and J. A. Peláez: Spaces of analytic functions of Hardy-Bloch type. - J. Anal. Math. 100, 2006, 53-81.

[18] Girela, D., and J. A. Peláez: Integral means of analytic functions. - Ann. Acad. Sci. Fenn. Math. 29, 2004, 459-469.

[19] Girela, D., and J. Peláez: Carleson measures, multipliers and integration operators for spaces of Dirichlet type. - J. Funct. Anal. 241, 2006, 334-358.

[20] Girela, D., and J. Peláez: Carleson measures for spaces of Dirichlet type. - Integral Equations Operator Theoary 61, 2006, 511-547.

[21] Hardy, G. H., and J. E. LitTlewood: Some properties of conjugate functions. - J. Reine Angew. Math. 167, 1932, 405-423.

[22] Hardy, G. H., and J. E. Littlewood: Some properties of fractional integrals II. - Math. Z. 34, 1932, 403-439.

[23] HeInz, E.: On certain nonlinear elliptic differential equations and univalent mappings. - J. Anal. Math. 5, 1956/57, 197-272.

[24] Ivashrovich, S., S. Pinchuk, and J. P. RosaY: Upper semi-continuity of the kobayashiroyden pseudo-norm, a counterexample for hölderian almost complex structures. - Ark. Math. 43, 2005, 395-401.

[25] PAvlović, M.: On Dyakonov's paper Equivalent norms on Lipschitz-type spaces of holomorphic functions. - Acta Math. 183, 1999, 141-143.

[26] Pavlović, M.: Introduction to function spaces on the disk. - Mat. Inst. SANU, Beograd, 2004.

[27] Pavlović, M.: Green's formula and the Hardy-Stein identities. - Filomat 23, 2009, 135-153.

[28] Pavlović, M.: Hardy-Stein type characterization of harmonic Bergman spaces. - Potential Anal. 32, 2010, 1-15.

[29] Rudin, W.: Function theory in the unit ball of $\mathbf{C}^{n}$. - Springer-Verlag, New York, Heidelberg, Berlin, 1980.

[30] SChiff, J. L., and W. J. WALKeR: A sampling theorem for a class of pseudoanalytic functions. - Proc. Amer. Math. Soc. 111, 1991, 695-699.

[31] SHI, J. H.: Inequalities for the integral means of holomorphic functions and their derivatives in the unit ball of $\mathbf{C}^{n}$. - Trans. Amer. Math. Soc. 328, 1991, 619-637.

[32] Stević, S.: Area type inequalities and integral means of harmonic functions on the unit ball. - J. Math. Soc. Japan 59, 2007, 583-601.

[33] Wirths, K. J., and J. XIAO: An image-area inequality for some planar holomorphic maps. Results Math. 38, 2000, 172-179.

[34] Yamashita, S.: Dirichlet-finite functions and harmonic majorants. - Illinois J. Math. 25, 1981, 626-631.

[35] ZHU, K.: Spaces of holomorphic functions in the unit ball. - Springer, New York, 2005. 